\title{
Componentes normativas de alta incidencia en la nueva morfología del Santiago Metropolitano: una revisión crítica de la norma de "Conjunto Armónico"
}

Elke Schlack Académica e Investigadora del Centro de Investigaciones Territoriales y Urbanas (CITU) de la Universidad Andrés Bello y Académica de la Escuela de Arquitectura y del Magíster de Proyecto Urbano de la Pontificia Universidad Católica de Chile.

Magdalena Vicuña Académica e Investigadora de la Escuela de Arquitectura, Escuela de Diseño y del Magíster de Proyecto Urbano de la Pontificia Universidad Católica de Chile.

RESUMEN | Mayor altura, mayor densidad habitacional y diferenciación tipológica respecto del entorno son las principales cualidades de los edificios que se construyen actualmente en el marco de la normativa chilena llamada "Conjunto Armónico". Considerando que esta normativa promueve la excepción morfológica y funcional de los proyectos respecto a su entorno, se sostiene que esta regulación podría constituir una manera de normar edificios excepcionales en la ciudad: aquello que hoy se conoce como "megaproyectos". Este artículo ilustra dos momentos en la evolución de esta norma. En ambos se puede visualizar la intención de constituir piezas de relevancia urbana. Se muestra la evolución de la norma y se ejemplifica a través de casos del periodo inicial, asociado a la visión composicional (1930-50), y de un segundo período, asociado a la visión funcionalista de la ciudad (1960-80). Se contrastan los objetivos asociados a estas dos etapas, con las cualidades que actualmente promueve la norma de Conjunto Armónico. Se reflexiona acerca de la pertinencia actual de la norma y se comentan las posibilidades que brinda hoy este instrumento para la gestión de piezas de relevancia urbana.

PALABRAS ClAVE | Morfología urbana, proyecto urbano, renovación urbana, espacio público.

ABSTRACT | Increased height, higher residential density and typological differentiation regarding the environment are the main characteristics of buildings that are currently constructed int eh context of the Chilean legal norm known as "Harmonic Set". Considering that this norm promotes the morphological and functional exception of such projects regarding their environment, it is maintained that this regulation could represent one way to regulate certain exceptional buildings in the city: those that today are known as megaprojects. This articule illustrates two moments in the evoluation of this legal norm. In both, the intent to form relevant urban archetypes can be clearly seen. It shows the evolution of this norm and exemplifies this process through cases from the initial period, associated with the compositional vision (1930-50), and a second period associated with the functionalist vision of the city (1960-80). The objectives associated with each period are compared and contrasted with the qualities that currently promote the legal norm of harmonic set. We reflect upon the pertienence of the norm, and discuss the possibilities that this instrument provides today for the creation of urban archetypes.

KEY WORDS | Urban morphology, urban project, urban renewal, public space 


\section{Introducción}

Las principales áreas metropolitanas de Latinoamérica están sufriendo importantes procesos de transformación, asociados a la expansión física, emergencia de nuevas centralidades e intensificación del territorio, entre otros fenómenos (De Mattos, 2002). Producto de dicho proceso de "metropolización expandida" (De Mattos, 2004), ciudades como São Paulo, México DF, Río de Janeiro, Buenos Aires, Santiago o Quito, hoy se constituyen como configuraciones urbano-regionales, cuyas inversiones tanto públicas como privadas han sido determinantes de los procesos de densificación de áreas centrales y creación de nuevos subcentros o nodos. A su vez, las políticas de liberalización y desregulación, pertenecientes al modelo de desarrollo capitalista (Castells) o urbanismo neoliberal (Theodore et al., 2008), han sido un factor determinante de dichas transformaciones urbanas.

En el contexto de la nueva arquitectura productiva global (Sassen, 2002), en el cual es primordial el progresivo posicionamiento de las ciudades latinoamericanas, se hace fundamental el análisis y reflexión sobre las normas urbanas y su rol en el presente contexto económico. En este artículo se hace referencia a la norma urbana chilena y, específicamente, a la norma de "Conjunto Armónico" y su aplicación en la ciudad de Santiago.

En la legislación urbana chilena se denomina "Conjunto Armónico" a aquella figura que permite aumentar la edificabilidad (metros cuadrados construidos totales), la densidad y la altura de un edificio, más allá de lo establecido en el instrumento de planificación local (Plan Regulador Comunal) ${ }^{1}$. La legislación vigente establece tres condiciones alternativas para que un proyecto pueda beneficiarse con dicha excepción: estar emplazado en un "terreno mayor" (más de $2.500 \mathrm{~m}^{2}$ o una manzana completa), estar destinado a equipamiento y estar localizado en, o colindante con, un terreno cuyos edificios estén declarados Monumentos Nacionales (ver Anexo 1). La mayoría de los proyectos desarrollados al amparo de esta norma durante la última década en la ciudad de Santiago cumplen, casi exclusivamente, con la condición de estar emplazados en un terreno mayor. Ejemplos de este último período son el edificio "Imago Mundi", el edificio "Isidora 3000", los edificios del sector "Nueva Las Condes", el conjunto de edificios de la "Sede Bellavista de la Universidad San Sebastián” con las tres torres de vivienda aledañas y los edificios del conjunto "Barrio lo Gallo", entre muchos otros.

Algunos de estos casos han llamado la atención de la comunidad. En la prensa de los últimos años se manifiesta una intensa discusión entre el sector público, organizaciones ciudadanas, arquitectos, urbanistas y gestores inmobiliarios. El tópico más relevante de esta discusión es el fuerte impacto que provocan los Conjuntos Armónicos en barrios residenciales de baja altura y baja densidad.

Un acucioso registro realizado recientemente sobre la evolución de esta norma (Alcaíno, 2008) permite construir la hipótesis sobre las etapas de transformación

1 Artículo 109 de la Ley General de Urbanismo y Construcciones (LGUC) (DFL 458 de 1976, actualizada por la Ley 20.389 D.O. 24.10.2009 y Artículos 2.6.4 al 2.6.19 de la Ordenanza General de Urbanismo y Construcciones (OGUC) (versión de los D.S. $\mathrm{N}^{\circ} 56$ y N 58 Vivienda y Urbanismo del 2009). 
que ha experimentado esta norma. Se sostiene que el Conjunto Armónico fue concebido como una norma para edificios singulares que cumplen un rol jerárquico en su entorno. Esta tesis será comprobada en dos momentos de la evolución de la norma: en la etapa 1930-1950, asociada a una aproximación morfológica del urbanismo, y en la etapa 1960-1980, relacionada a una visión funcional de la ciudad. Bajo el supuesto de que las normas son portadoras de imagen ciudad y reflejan el proyecto político y social de una sociedad, se muestra cuál fue el espíritu inicial de esta norma y cómo fue evolucionando su conceptualización, formulación y aplicación hasta la actualidad.

El objetivo es aportar al debate con información técnica y así abrir preguntas acerca del sentido que tuvo y tiene esta norma de excepción en la conformación de la espacialidad urbana y su potencial como instrumento de renovación urbana a partir de piezas estratégicas en la ciudad.

2 La noción de "Conjunto Armónico" en el Santiago de 1930 a 1950: una aproximación estética del urbanismo

\subsection{La regulación del Conjunto Armónico en la Ordenanza Local de Edificación para la Comuna de Santiago de 1939}

Si bien la Ley y Ordenanza General de Construcciones y Urbanización de 1936 se refiere a los conceptos "conjunto", "armónico" y "estético", siempre relacionados al aspecto exterior de los edificios, la primera mención explícita del concepto "Conjunto Armónico" aparece en la Ordenanza Local de Edificación para la Comuna de Santiago y Ordenanzas Especiales de 1939. El concepto está basado en la Ley de 1936 y en las propuestas del arquitecto austríaco Karl Brunner para Santiago. Estas conceptualizaciones también fueron recogidas en otros Planes Reguladores de la época, como el de Providencia (1944) y el de San Miguel (1951).

En efecto, la Ley General de Construcciones y Urbanización de 1936 hace alusión a dichos conceptos en el párrafo correspondiente a la altura y el aspecto exterior de los edificios:

Los propietarios, sin perjuicio de lo dispuesto en los artículos 49 y 50 (referidos a altura y antejardín), podrán adoptar en sus edificios los tipos de arquitectura o de ornamentación que estimen convenientes, siempre que su conjunto o detalle no fueren contrarios a la estética.

Estas referencias transversales a la armonía arquitectónica y a la unidad estética del conjunto son un mecanismo para el diseño urbano y la composición arquitectónica de la ciudad, y no constituyen una norma de excepción tal como se conoce hoy.

Por su parte, la Ordenanza de Santiago de 1939, en su Capítulo referido al ornato público, estipula que "las fachadas no podrán recibir pinturas o estucos de diferentes colores que perturben la armonía del conjunto" (Artículo 479); que "los Municipios podrán fijar alturas mínimas o máximas o ambas a la vez, para los edificios que se construyan en distintas zonas o con frente a determinadas calles, plazas o avenidas en razón de su carácter o efecto estético del conjunto de la edificación” 
(Artículo 480) y, finalmente, que "los Municipios, con aprobación especial del Presidente de la República, podrán establecer en cada caso, como obligatorias para una calle, plaza o avenida o para una cierta extensión de ésta, la adopción de determinado estilo en las fachadas y alturas correlativas de piso en los edificios, con el fin de obtener un efecto armónico con el conjunto de la edificación” (Artículo 484).

El concepto de "Conjunto Armónico" aparece como tal en el Artículo 11:

Todos los estudios de Arquitectura que se proyectan para ser realizados en el sector para el cual rige la presente Ordenanza, deberán ser sometidos a una aprobación del Anteproyecto (...) con el fin que en su totalidad constituyan un conjunto armónico...

Es posible observar que en la Ordenanza de Santiago, el concepto de Conjunto Armónico tiene básicamente tres consideraciones:

\section{a El conjunto armónico como mecanismo para la conservación de una pieza urbana y su condición arquitectónica existente}

El concepto de "Conjunto Armónico" se refiere, en primer término, a aquellos conjuntos de edificaciones que es necesario preservar. Por ejemplo, la Ordenanza considera "conjunto armónico" el Sector Especial de la Plazuela del Teatro Municipal (San Antonio con Agustinas). De acuerdo al Catastro de Santiago (1939)2 , por tratarse de la conservación de los elementos que constituían la plazuela, no se establecían condicionantes adicionales para este tipo de conjunto. Justamente, las tres propiedades correspondientes a las viviendas construidas en 1901 por Francisco Subercaseaux (del arquitecto Miguel Ángel de la Cruz Labarca) ${ }^{3}$, así como la plazoleta y el conjunto de elementos y espacios arquitectónicos que enfrentan dichos edificios, serán posteriormente designados como Monumento Nacional ${ }^{4}$.

Para el caso del sector comprendido entre las calles Estado, Santo Domingo, San Martín y la Alameda (y la acera oriente de la calle Estado), la Ordenanza establecía que se debía mantener una "altura establecida como conjunto armónico".

La Ordenanza de 1939 también establece como conjunto armónico las manzanas comprendidas entre las calles (a) Agustinas, Estado, R. Nieto y Matías Cousiño; (b) R. Nieto, Estado, Moneda y Matías Cousiño y (c) Moneda, Estado, M. Cruchaga y Matías Cousiño (Figura 1), además de "las manzanas chicas ubicadas en Teatinos esquina de Compañía y Teatinos esquina de Huérfanos, las que se conservarán como conjuntos armónicos" (Figura 2).

2 Catastro realizado por la Municipalidad de Santiago en 1939. Fuente: Proyecto FONDECYT N ${ }^{\circ} 1085253$ “Santiago 1910. Construcción planimétrica de la ciudad pre-moderna. Transcripciones entre el fenómeno de la ciudad física dada y la ciudad representada”. Investigador Responsable: José Rosas. Co-Investigadores: Germán Hidalgo, Wren Strabucchi e Italo Cordano.

3 En la década del 30, dos de las viviendas correspondían al Círculo Francés y el Club Militar.

4 Dcto: N159/73.

5 Correspondiente al Conjunto Los Lirios 1 y 2.

6 En este caso, se establece una altura de 25 metros para toda la manzana, con excepción de los edificios que se conservan como conjunto armónico. 
FIGURAS 1 Y 2 | Manzanas establecidas como conjunto armónico en la Ordenanza de 1939
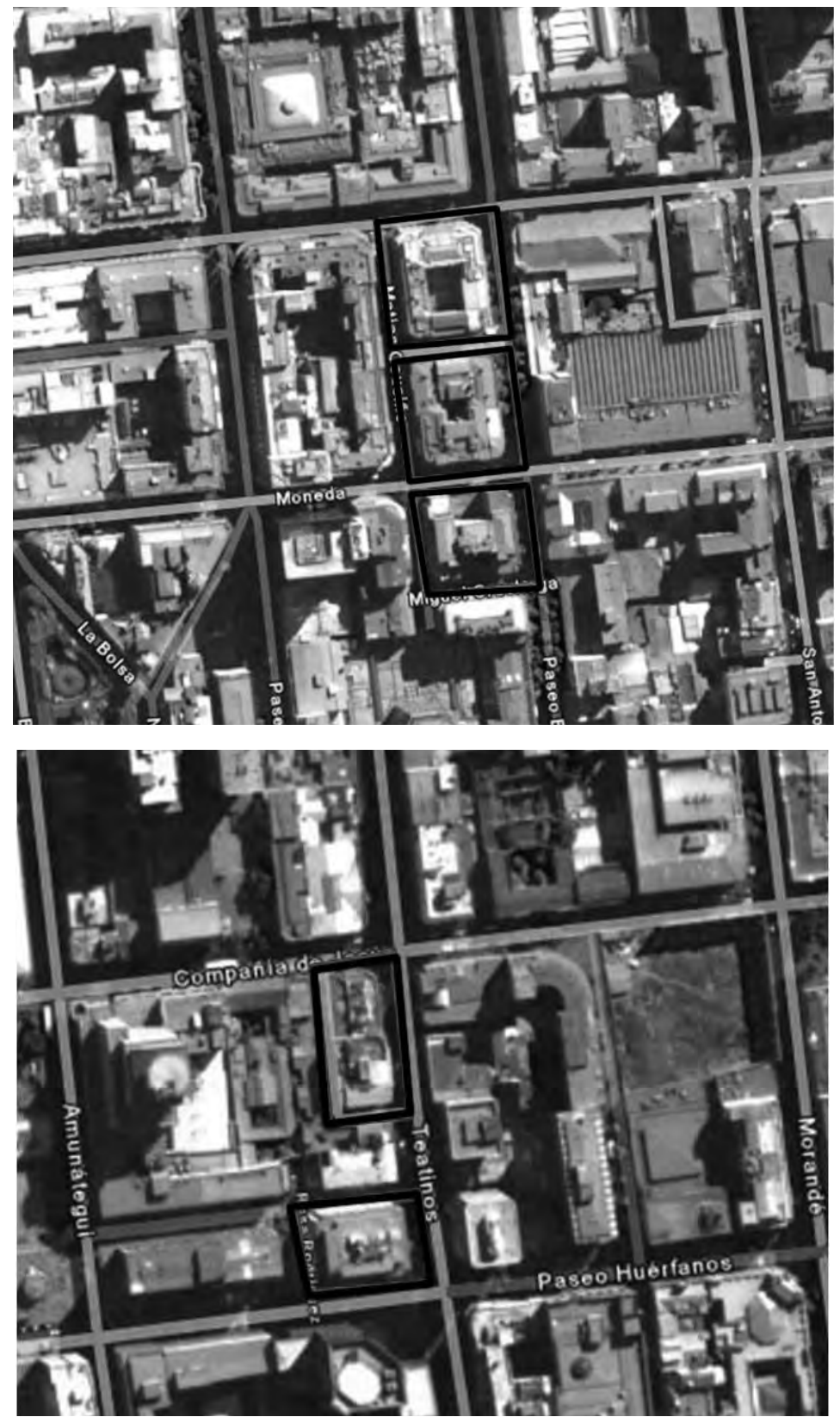
De esta manera, la unidad de aplicación del conjunto armónico son parcelaciones de la manzana, producto de la conformación de piezas arquitectónicas relevantes y necesarias de conservar, como por ejemplo, el edificio del Banco Español.

\section{$b$ El conjunto armónico como mecanismo para el desarrollo de un sector con nuevas edificaciones que constituyan una unidad arquitectónica}

La necesidad de componer arquitectónicamente la ciudad, planteada inicialmente por Brunner, dará una segunda interpretación al concepto de "Conjunto Armónico".

En el caso del sector comprendido entre la Plaza de Armas, la calle Puente, la Avenida Balmaceda y calle 21 de Mayo (Figura 3), la Ordenanza de 1939 estableció que "las líneas de edificación que en el Plano $\mathrm{N}^{\circ} 1$, letra $\mathrm{A}$, se dan con carácter informativo para el conjunto designado con letra $\mathrm{D}$, estarán sujetas al estudio especial que se realice para él, el cual se proyectará de manera que constituya un solo conjunto armónico". Para este sector se establece también que "los elementos que se construyan sobre la losa cubierta de los últimos pisos, deberán ser proyectados en forma armónica con el edificio y el conjunto total" y que "todos los estudios de arquitectura que se proyecten (...), deberán ser sometidos a una aprobación del anteproyecto por el Departamento de Obras de la Municipalidad, el cual podrá rechazarlos o introducir modificaciones, con el fin que en su totalidad constituyan un conjunto armónico (...)"8

Cabe señalar que para este sector se establecía también una zonificación de uso comercial en el primer piso y la construcción de galerías comerciales interiores con acceso desde las calles9. En este caso, el conjunto armónico constituía una herramienta de diseño urbano que buscaba una relación legible entre la Plaza de Armas y el Mercado Central, a partir de la unificación visual de las manzanas que separan dichos elementos.

Artículo $10^{\circ}$, Ordenanza Local de Edificación para la Comuna de Santiago y Ordenanzas Especiales, 1939, p. 13.

Artículo $11^{\circ}$, p. 13 de la Ordenanza de Santiago. Respecto de esta consideración, es interesante señalar que el Plan Regulador de Providencia de 1944 señala en su Artículo 26, Capítulo VI, referido a las exigencias de aspecto exterior, en relación a la zona especial del Parque Providencia “(...) la edificación en la calle Román Díaz entre Providencia y Costanera, estará de acuerdo con las líneas arquitectónicas del Plano de conjunto que aprobará la I. Municipalidad para este sector". También se hace alusión a que las nuevas edificaciones en los sectores de Plaza Los Leones, Plaza Canal San Carlos y Plaza Pedro de Valdivia, los cuales debían guardar uniformidad arquitectónica con las propiedades vecinas. Por otro lado, el Plan Regulador de San Miguel también utiliza la figura de conjunto armónico para definir la morfología de un determinado sector. En efecto, la altura mínima reglamentada no rige para una serie de sectores definidos en el Plan Regulador y se hace referencia explícita a "bloques armónicos", los cuales debían constituir unidades arquitectónicas.

9 La altura del costado poniente de la calle Puente era de 30 metros, retornando sus fachadas en las esquinas entre 15 y 20 metros. La altura máxima permitida era, en términos generales, de 41,50 metros y la altura del primer piso comercial era de 6.35 metros. La altura del segundo piso comercial era de 3.25 metros y 2.85 metros entre pisos para el resto de los pisos. 
FIGURA 3 | Sector definido como conjunto armónico por la Ordenanza de 1939

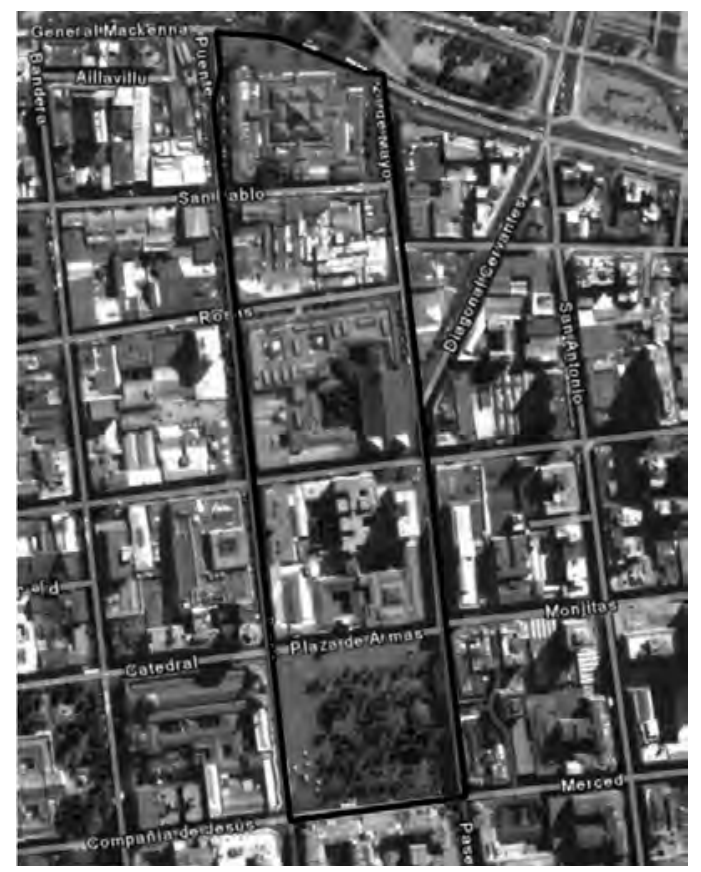

FUente Elaboración propia en base a Google Earth 2010.

c El conjunto armónico como norma de excepción para aquellos sectores que conforman una unidad

Esta tercera consideración constituye quizás la primera alusión a la norma de Conjunto Armónico como norma de excepción, la que posteriormente también será aplicada en los Planes de Providencia y San Miguel. La Ordenanza de Santiago estableció que, bajo el concepto de "Blocks armónicos de edificación”, el Municipio podía

(...) autorizar para algunos lotes un aprovechamiento mayor que el fijado para la zona respectiva a condición de que en el conjunto no se sobrepase la cifra correspondiente a la zona, ni se perjudiquen la iluminación y ventilación de las habitaciones. Igualmente, dicha Dirección podrá otorgar en los casos a que se refiere este artículo y cuando del estudio de conjunto ello resultare conveniente, una alteración en las alturas y en los tipos de edificación ${ }^{10}$.

10 En su Capítulo VI, Artículo 48, la Ordenanza señala que "A base de un plan armónico de repartición y edificación para un conjunto de construcciones o para una población, la Dirección de Obras Municipales podrá autorizar para algunos lotes un aprovechamiento mayor que el fijado para la zona respectiva a condición de que en el conjunto no se sobrepase la cifra correspondiente a la zona, ni se perjudiquen la iluminación y ventilación de las habitaciones.

Igualmente, dicha Dirección podrá otorgar en los casos a que se refiere este artículo y cuando del estudio de conjunto ello resultare conveniente, una alternación en las alturas y en los tipos de edificación a los que se refieren los capítulos II y IV (Estas disposiciones no regirán para las zonas I y II)". 
El incentivo estaba relacionado con la tipología edificatoria, mediante el beneficio de mayores alturas y flexibilidad en las formas de agrupamiento de la edificación. En este sentido, el Artículo $11^{\circ}$ de la Ordenanza exceptuaba de las disposiciones de altura a aquellos sectores donde exigía alturas uniformes para varios edificios que forman un conjunto plástico determinado ${ }^{11}$.

La Ordenanza establecía para el Sector de la Plaza Argentina, frente a la Estación Central, que la altura de los edificios debía ser uniforme de catorce metros (4 pisos aproximadamente). "Sin embargo, la Dirección de Obras Municipales podrá autorizar una mayor altura siempre que se formen blocks armónicos, cuyo conjunto no perturbe la armonía del total".

De manera similar, las ordenanzas de Providencia y San Miguel hacen alusión al concepto de "Conjunto Armónico" como norma de excepción. En el caso de Providencia, se establece que la DOM puede autorizar una mayor altura “(...) siempre que se trate de cuerpos retirados del plano vertical de fachada y siempre que se cumpla con el Art. 482 de la Ordenanza General de Construcción y Urbanización, constituyendo esta mayor altura un conjunto compuesto en armonía plástica con la edificación vecina”. Esta Ordenanza también permitía otorgar permisos de edificación con frentes desde 8 metros (menores al mínimo exigido en la comuna), siempre y cuando la edificación conformara grupos o blocks de edificación continua proyectados como conjuntos plásticamente homogéneos.

Por su parte, la Ordenanza de San Miguel establecía que la Dirección de Obras podía autorizar mayor número de pisos cuando se tratara de bloques armónicos o cuando la mayor altura correspondiera a "un conjunto plástico estructurado literalmente"12.

Amparados en el Artículo 51 de la LGCU, estos instrumentos señalaban que en el caso de que existiera alguna divergencia entre la Municipalidad y el propietario respecto de los valores estéticos de una edificación, se convocaría a un jurado compuesto por tres arquitectos (uno designado por el Alcalde, otro por el propietario y un tercero por el Director General de Obras Públicas). Este punto vuelve a confirmar el rol que tenía la norma como un instrumento de diseño urbano, en el cual, consecuentemente con los planteamientos de Brunner, las condiciones espaciales y arquitectónicas eran determinantes para su condición de conjunto armónico.

11 Artículo $11^{\circ}$, Ordenanza Local de Edificación para la Comuna de Santiago y Ordenanzas Especiales, 1939, p. 15.

12 En el Artículo 8, Capítulo IV de la Ordenanza Local de Providencia de 1944, se establece que "la DOM podrá autorizar una mayor altura que la contemplada en el cuadro anterior siempre que se trate de cuerpos retirados del plano vertical de fachada, siempre que se cumpla con el Art. 482 de la Ordenanza General de Construcción y Urbanización, constituyendo esta mayor altura un conjunto compuesto en armonía plástica con la edificación vecina (...)", p. 12. A su vez, en el Artículo 24, Capítulo V, se establece que "se puede otorgar permisos de edificación con frentes desde 8 mts en grupos o blocks de edificación continua proyectados como conjuntos homogéneos plásticamente conformados y cuya extensión total no sea superior a $45 \mathrm{mts}$.(...)", p. 13.

En la Ordenanza correspondiente a la Comuna de San Miguel (1951) el concepto de conjunto armónico se establece no sólo para hacer referencia a unidades arquitectónicas, sino que también se constituye como una norma de excepción: "La Dirección de Obras Municipales podrá autorizar en determinados casos mayor número de pisos (...), cuando se trate de bloques armónicos o cuando la mayor altura corresponda a un conjunto plástico estructurado literalmente" (Artículo 93). 
Finalmente, destaca el Artículo 17 de la Ordenanza de Santiago, el cual señalaba que se podían construir pisos adicionales sobre la altura máxima permitida por la norma, siempre y cuando se preservara la iluminación de las propiedades vecinas; no quedaran muros cortafuegos altos en manzanas con edificación baja; el aumento de los vehículos estacionados no dificultara el tránsito; y en la cuadra dominaran edificios de una altura superior a la máxima establecida en la Ordenanza, construidos con anterioridad a la fecha en que ésta fuera puesta en vigencia. En esta Ordenanza es posible encontrar la primera versión de un tipo de normativa más flexible, en la cual se establece un mayor beneficio edificatorio, siempre y cuando se cumpliera con determinadas condiciones, tal como se establece en la normativa vigente de Conjunto Armónico.

\subsection{El contexto: paradigmas e instrumentos y la propuesta de Karl Brunner}

No es posible analizar esta primera etapa de conceptualización del "Conjunto Armónico” sin considerar los planteamientos del arquitecto Karl Brunner (Almandoz, 2007) y su propuesta para Santiago, específicamente la propuesta para el Barrio Cívico en el entorno de La Moneda y la apertura del eje de la Avenida 12 de Febrero, hoy Avenida Bulnes, en la década de los años 30 (Brunner 1932, Brunner 1939).

Tal como señala Gurovich, Brunner constituye el más importante representante en Latinoamérica del arte urbano racionalista y contextualizado (Almandoz 2007, p. 9, Gurovich 1996, pp. 8-13) y sus planteamientos empapan el pensamiento de arquitectos y urbanistas de la época, influenciando también la incipiente legislación urbana y el nacimiento de la norma de Conjunto Armónico ${ }^{13}$.

Brunner planteó, a partir de una aproximación más científica del urbanismo, una idea integral de la planificación urbana, en la cual la morfología urbana existente era tan relevante como los aspectos técnicos, económicos y sociales que determinan el desarrollo de la ciudad (Pérez y Rosas, 2002).

Para Brunner, el urbanismo tiene la capacidad de crear un conjunto armónico en el contexto urbano:

el urbanismo actual considera en primer término el aspecto cultural y de higiene social de su misión relacionada con los problemas político-económicos, en seguida el aspecto técnico-ingenieril de los problemas y las necesidades que crea, y en tercer lugar el aspecto artístico-estético, que se esmera en crear un conjunto armónico en el cuadro de la ciudad (Brunner 1930a, p. 12).

Estos postulados evocarán los del Movimiento "City Beautiful”, el cual planteaba que la ciudad es una conjunción entre los valores institucionales y la monumentalidad de las construcciones: "la arquitectura como centro de la conformación de los espacios públicos" (Peterson, 2002). Así, el concepto de "Conjunto Armónico" surge desde la noción de una ciudad que se construye a partir de la arquitectura y su capacidad de representación institucional.

Es posible constatar que en el discurso de Brunner aparece el concepto de "Conjunto Armónico" como parte de las funciones del urbanismo moderno, constitu-

13 De acuerdo a Gurovich, entre éstas se encuentran la Ley 5604 (1935), la cual autorizaba al Departamento de la Habitación expropiar terrenos; la Ley 5950 que creaba la Caja de Habitación Popular, la Ley 6071 (1937), llamada "de propiedad horizontal" o de "venta por pisos y departamentos" y el DS 3424 que aprobaba el Plan Seccional del Sector Barrio Cívico de Santiago. 
yendo el espacio público el principal elemento articulador de la institucionalidad y monumentalidad de la forma urbana (Aguirre, 2005):

(...) para llegar a ser una ciudad de carácter arquitectónico, es necesario establecer las relaciones entre las calles y espacios libres con sus edificios monumentales (...) El sistema de cuadras (manzanas) de la ciudad de Santiago de Chile (...) trae como consecuencia que casi todos los edificios públicos no queden bien emplazados para cumplir con el rol decorativo y de representación dentro de la ciudad" (Brunner 1930b, pp. 265).

Consecuentemente, Brunner sugerirá una serie de formas de dividir, modificar y enriquecer la manzana colonial, con el fin de multiplicar el frente comercial y proveer de nuevos espacios públicos, sin que ésta pierda su condición de conjunto armónico.

Este planteamiento se refleja en la primera consideración del conjunto armónico anteriormente señalada (el conjunto armónico como mecanismo para la conservación de una pieza urbana y su condición arquitectónica existente). A su vez, Brunner propuso mecanismos para reglamentar la zonificación de usos y las condicionantes de la edificación que determinaban la unidad arquitectónica de las construcciones y que propiciaban un efecto estético en base a la relación entre el espacio público y el espacio privado ${ }^{14}$. En este sentido, el conjunto armónico corresponderá a uno de los mecanismos que propiciaban dichos objetivos morfológicos en la ciudad ${ }^{15}$. La propuesta de Brunner iba en una dirección diferente a los planteamientos de Le Corbusier y los CIAM, los cuales se basaban más bien en el concepto de tabula rasa.

La necesidad de resguardar el aspecto de conjunto y armonía arquitectónica impulsada por Brunner se materializará en el Plan Oficial de Urbanización ${ }^{16}$ y la mencionada Ordenanza Local de Santiago de 1939, la cual regulaba no sólo la intensidad de la edificación a partir de alturas máximas, ocupación de suelo y subdivisiones prediales, sino que también establecía detalladas consideraciones de tipo estético y de composición arquitectónica, tales como materialidad, color y volumetría de las edificaciones.

\subsection{El Paseo Bulnes: una operación de conjunto armónico}

Si bien la Ordenanza de Santiago de 1939 no lo explicita como conjunto armónico, es posible conjeturar que la operación del Paseo Bulnes decanta el espíritu de este concepto. El Paseo Bulnes es producto de la combinación de un modelo de ciudad, una normativa urbana orientada a una precisa definición morfológica de las edificaciones y mecanismos innovadores de gestión.

14 En efecto, Brunner señalará que las "disposiciones de esta naturaleza fijan la consistencia corpórea de la manzana, dándole siluetas determinadas en sus perfiles, lo que es un peligro para el efecto estético que debe producir una ciudad" (Brunner, 1929).

15 En su propuesta para el Plan de Santiago, Brunner recomendaba que las calles no condujeran en línea recta a través de los terrenos en una extensión mayor a 250-300 metros; que las calles residenciales tuvieran dirección norte-sur y que las de dirección oriente-poniente tuvieran mayor ancho (ambas consideraciones para optimizar las condiciones de asoleamiento); que las edificaciones en altura se escalonaran hacia el centro de la manzana y que la altura máxima en el centro no superara los 30 metros, siempre y cuando la proporción entre el ancho de la calle y la altura de los edificios no superara el 1 a 1 y $1 / 2$ y que se tomara en cuenta la situación desfavorable en quedaban los edificios con orientación oriente-poniente (esto se solucionaba abriendo parte de la manzana en forma tal, que dejara entrar el sol desde el norte, disponiendo calles divisorias interiores de Norte a Sur).

16 En 1939 se aprueba el Plan Oficial de Urbanización de la Comuna de Santiago, para regular la edificación en altura, aprovechar al máximo los terrenos y subdivisiones permitidas, clasificar la zona industrial y desarrollar un Plan de vialidad y áreas verdes. 
A su vez, este proyecto constituirá un referente para el desarrollo posterior de la figura del Conjunto Armónico en la Ordenanza General de Urbanismo y Construcciones ${ }^{17}$, ya que ejemplificó el potencial que tenían las normas referidas a conceptos estéticos y las normas de excepción a los Planes Reguladores.

El proyecto para el Barrio Cívico y el Paseo Bulnes se inició en el gobierno de Carlos Ibáñez del Campo y se concretó en el transcurso de casi cuatro décadas ${ }^{18}$. El proyecto, terminado por el arquitecto Carlos Vera Mandujano, consistía en la apertura de la Avenida Central, desde el Palacio de la Moneda hasta la Plaza Almagro, a partir de un gran espacio público flanqueado por edificios bloque de 39 metros de altura ${ }^{19}$.

El proyecto demostrará una drástica separación entre el espacio privado y el espacio público y una lógica racional en la división de funciones o actividades: el comercio y las oficinas se ubicaban en el primer piso y las viviendas en los pisos superiores ${ }^{20}$.

La propuesta de Brunner decantaba las ideas iniciales de Camilo Sitte, quien visualizaba el urbanismo como un problema estético y valoraba el espacio público a partir de su proyección como espacio unitario y legible con un rol determinante en la composición de la morfología urbana (Sitte, 1965).

Tal como señala Rosas, la propuesta del Paseo Bulnes se refuerza con la diferencia existente entre las viviendas que enfrentan la Avenida Central y aquellas insertas al otro lado de la manzana. En términos generales, los edificios organizan la planta con los recintos "nobles" hacia la calle y los servicios "ciegos" hacia el interior (Rosas, 2009). De esta manera, el conjunto armónico se refleja en un espacio privado que se homogeneiza mediante una arquitectura coherente que refuerza la legibilidad del espacio público.

Posteriormente, el Plan General de Urbanización para Santiago estableció una normativa especial para el Barrio Cívico que determinaba la homogeneidad y armonía estética y morfológica de las nuevas edificaciones que se construirían en torno a La Moneda, su espacio público y el Eje Bulnes ${ }^{21}$.

El modelo de gestión colectiva coordinada por el Ministerio de Obras Públicas, con una activa participación del sector privado y basado en la figura de captura de plusvalías, fue tan relevante como las condiciones morfológicas propuestas ${ }^{22}$.

17 Esto lo confirma la entrevista al arquitecto Amador Brieva realizada por las autoras y citada por Alcaíno (2008).

18 Los objetivos iniciales del proyecto para el Paseo Bulnes consistían en otorgar a Santiago un eje de salida hacia al sur que conectara con la Carretera Panamericana y con los caminos a San Bernardo y Santa Rosa (lo que nunca se concretó). A su vez, se buscaba generar una nueva centralidad, mediante la descongestión del centro y la creación de un nuevo sector comercial para la ciudad que, a partir del aumento de la oferta comercial, permitiera abaratar los costos de los locales.

19 Tal como se señala en el artículo “Avenida General Manuel Bulnes”, el proyecto final se realiza mediante una modificación al Plano Oficial de Urbanización de Santiago del año 1945. Esta modificación afectaba el trazado de la Avenida 22 de Febrero en toda su extensión, de la calle Gálvez hasta 10 de Julio, de la manzana comprendida entre las calles Inés de Aguilera, Gálvez, Nataniel y Eyzaguirre y manzanas adyacentes a ella y a la Gran Avenida Oriente-Poniente que consulta el Plano Oficial de Urbanización entre el templo de los Sacramentinos y el Palacio Cousiño (Revista Arquitectura y Construcción, 1945, pp. 53-60).

20 Al respecto ver Rosas, J. (2009).

$21 \mathrm{Al}$ respecto ver Artículo 10 Ordenanza Local de Edificación de la Comuna de Santiago y Ordenanzas Especiales 1939. Sector Avenida General Bulnes y Sector Sacramentinos.

22 El modelo de gestión para el Paseo Bulnes se fundamentó en la figura de captura de plusvalías, tal como se implementaron proyectos como el Parque Bustamante y la Avenida Diagonal Oriente, los cuales constituyen ejemplos de inversión pública en infraestructura y vialidad, con la finalidad de producir un aumento de plusvalías y un mejoramiento general de la imagen urbana. 
FIGURA 4 | El Proyecto inicial para el Paseo Bulnes. Vista hacia el sur desde La Moneda

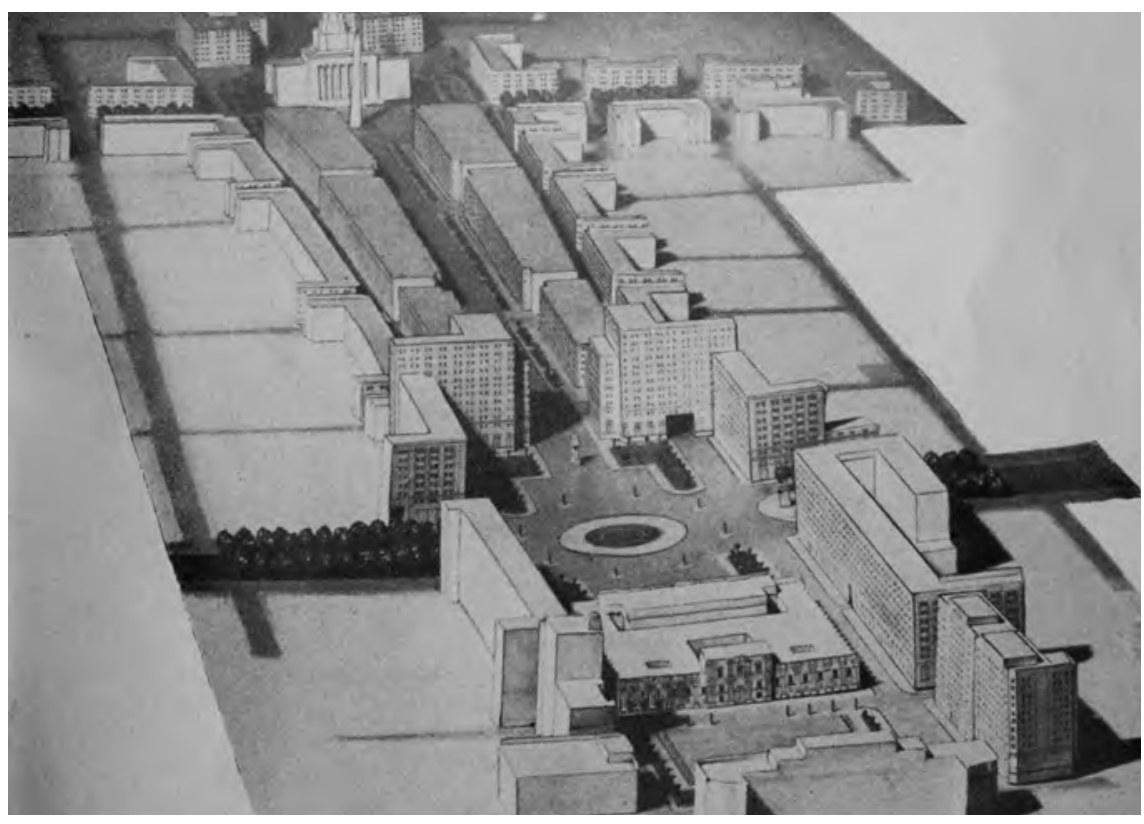

fuente Revista Areuitectura y Construcción 1945, p. 57.

El sector privado fue incentivado mediante créditos y exenciones tributarias ${ }^{23}$, bajo la coordinación de una Comisión Especial del Ministerio de Obras Públicas. La acción del MOP como agente coordinador fue fundamental, ya que persuadió a los propietarios para que cedieran los fondos de los sitios con la expectativa de una valorización de sus propiedades, como una forma de recuperación anticipada de plusvalías (Cáceres, Sabatini, 2001). Por su parte, el Estado estaba a cargo de comprar los terrenos destinados a las calles consideradas en el nuevo trazado ${ }^{24}$.

23 Tal como se señala en "Avenida General Manuel Bulnes", los incentivos tributarios aplicados otorgaban a todos los propietarios que desarrollaran proyectos de acuerdo al nuevo trazado y normativa propuestos en el Plan, la exención total del pago de contribuciones durante 10 años y la exención del 50\% de este impuesto durante los siguientes cinco años (durante un plazo de 3 años una vez aprobada la ley). Por otro lado, los propietarios que no desarrollaran nuevos proyectos tendrían una sanción de un impuesto territorial adicional en base al aumento del valor del inmueble producto del potencial de edificación que daban las exigencias de la nueva ordenanza (Revista Arquitectura y Construcción, 1945, pp. 53-60).

24 Las expropiaciones debían limitarse a lo estrictamente necesario para lograr la modificación del trazado de la calle Gálvez y la formación del Parque Almagro. En los casos que no se hacía posible la edificación porque quedaban retazos de terrenos, se autorizaba su venta directa a sus dueños o a los propietarios colindantes a un precio no inferior al valor de la expropiación. 
FIGURA 5 | Croquis de tributación que sostenía la captación de plusvalías. Las propiedades más cercanas al Paseo Bulnes debían aportar con mayores impuestos, ya que se suponía que adquirían mayor plusvalía

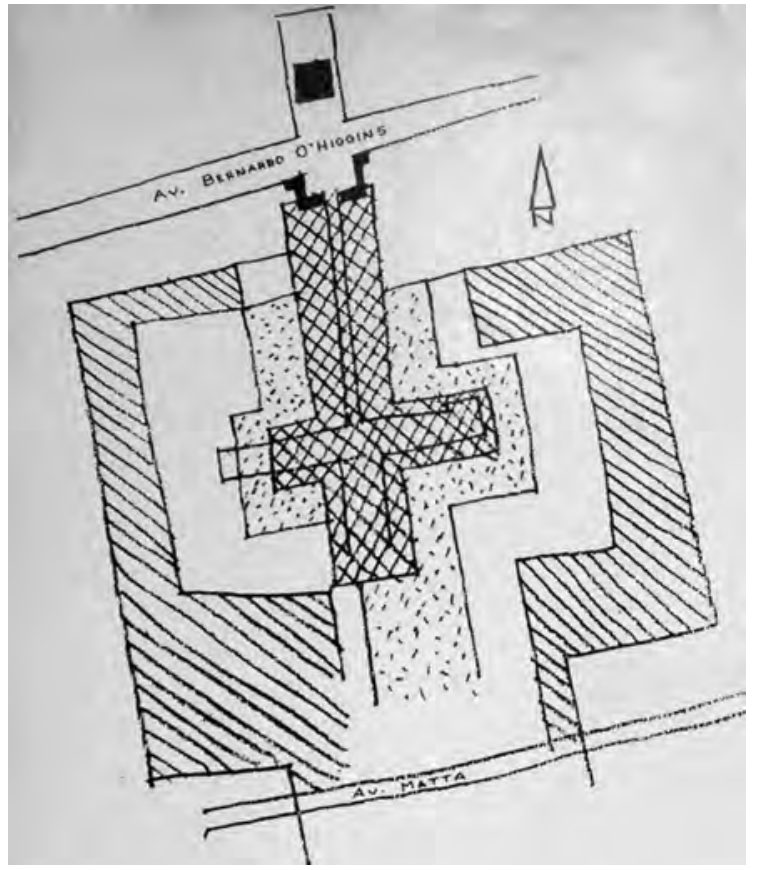

FUente Revista ArQuitectura y Construcción, 1945, P. 58.

El MOP realizó las obras de urbanización y mejoramiento del espacio público mediante créditos, los cuales, junto con las expropiaciones, se debían pagar a través de la venta de terrenos sobrantes y de los impuestos a los propietarios que no edificaban en sus terrenos. Estas obras y expropiaciones también se financiaron a través de un impuesto a la plusvalía, previamente implementado en la gestión del Parque Bustamante. Este impuesto especial se aplicaba a las propiedades que resultaban beneficiadas con las obras realizadas tanto por el Estado como por el sector privado (Revista Arquitectura y Construcción, 1945, pp. 53-60).

Tal como señalan Cáceres y Sabatini, la gestión de este proyecto fue exitosa porque se disponía de un fondo especial de financiamiento y porque fue gestionado por un único agente coordinador: el Ministerio de Obras Públicas, el cual le dio primera prioridad (Cáceres, Sabatini, 2001).

\subsection{Comentarios conclusivos acerca del sentido de la norma en esta etapa}

El "Conjunto Armónico" aparece primeramente en los planteamientos de Karl Brunner como un concepto relacionado al rol artístico y estético que se daba al urbanismo en la década del treinta. El urbanismo de Brunner decantó en los instrumentos legales de la época. Los mecanismos normativos de la Ley General, y más 
explícitamente de la Ordenanza de Santiago de 1939, valoraron la morfología urbana existente, representando una noción de ciudad que se construye a partir de su arquitectura. En la etapa de origen de la norma aparece el conjunto armónico como norma de excepción, otorgando mayores alturas y la posibilidad de construir nuevas tipologías arquitectónicas cuando las nuevas edificaciones conformaran una unidad.

El Conjunto Armónico es originalmente una norma de diseño urbano, un mecanismo que resguarda la conservación del patrimonio y que potencia la conformación de nuevos edificios que constituyen unidades arquitectónicas, a partir de una cuidada relación entre el espacio público y el espacio privado. En su origen, el Conjunto Armónico constituye un instrumento para regular la generación de piezas urbanas relevantes que cumplen un rol estratégico en su contexto urbano.

\section{Conjunto Armónico: un concepto de la planificación funcionalista}

\subsection{La regulación del Conjunto Armónico en la Ley y Ordenanza General de Urbanismo y Construcciones entre 1960 y 1980}

La Ley General de Urbanismo y Construcciones, en sus versiones de 1953 y 1960 regula, como principal fuente jurídica, el conjunto armónico en esta etapa. El instrumento declara de manera explícita el objetivo de la planificación urbana, estableciendo que "las experiencias y progresos del urbanismo moderno" están presentes en la ley ${ }^{25}$. Se disponen en ella prohibiciones relativas al "uso del suelo, la vialidad, los servicios públicos, la edificación (...), condiciones de higiene, de seguridad" y se pretende "obtener un aprovechamiento racional de los recursos de la comunidad ${ }^{26 \text { ". }}$

En el Artículo 95 de la versión de 1963 de dicha ley se menciona por primera vez la figura de "Conjunto Armónico" en una fuente jurídica de nivel nacional ${ }^{27}$. Posteriormente, en 1970, se define en el artículo 480 al Conjunto Armónico como "agrupamiento de diversas construcciones o edificios que estén relacionados de tal manera que constituyan una unidad espacial propia e identificable como tal ${ }^{28}$. Destaca la noción de una pieza "identificable como tal", conceptualización que sería complementada en 1976 con la siguiente definición: "Conjunto Armónico" son "aquellas agrupaciones de construcciones que (...) estén relacionadas entre sí, de tal manera que constituyan una unidad espacial propia, distinta del carácter general del barrio o sector"29. Con esto se le concede protagonismo al conjunto armónico, no sólo por constituir un conjunto, sino también por la posibilidad de diferenciarse del entorno.

La reglamentación específica de las condiciones y premios de la normativa de Conjunto Armónico se formula en detalle en la Ordenanza General de Urbanismo y Construcciones recién en 1970. En primer lugar, las condiciones exigidas se refieren a cualidades de uso y de tamaño predial. Conjuntos Armónicos pueden 
ser aquellos proyectos cuya "construcción corresponda a un edificio de equipamiento comunitario, como un mercado, gimnasio, templo, teatro, hospital, etc.”, o bien aquellos que constituyan "construcciones emplazadas en terrenos superiores a $2.500 \mathrm{~m}^{2}$ y $50 \mathrm{~m}$ de frente, o que representen un $33 \%$ de la manzana” ${ }^{30}$.

En segundo lugar, se establecía la siguiente condición: los proyectos acogidos a la norma de Conjunto Armónico debían "disponer un mínimo de $6 \mathrm{~m}^{2}$ de espacio común libre por habitante a nivel de terreno o en terrazas ${ }^{31}$ ”. Esta exigencia permaneció en la Ordenanza General hasta 1992, cuando se suprime ${ }^{32}$.

FIGURA 6 | Condición de dimensión, exigencia sustancial del “Conjunto Armónico”

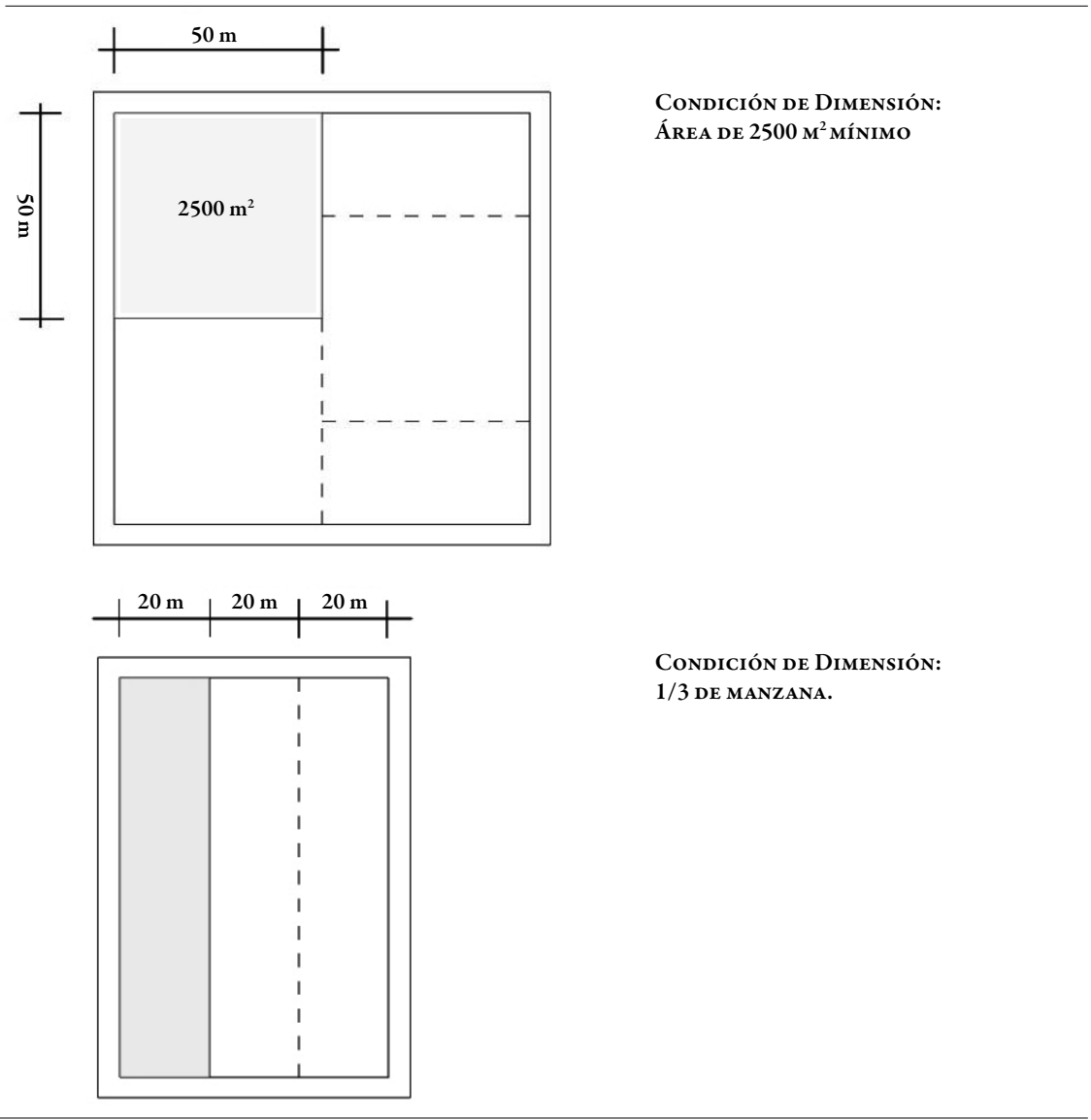

FUENTE ELABORACIÓN PROPIA 2010. 
Al mismo tiempo, se establece en la Ordenanza General de 1970 el beneficio con que se premia el conjunto armónico: el aumento del $50 \%$ de la densidad establecida por el Plan Regulador Comunal respectivo y la liberación de normas urbanísticas como altura, distanciamientos, ocupación de suelo y coeficiente de constructibilidad. El beneficio de constructibilidad se modifica en las sucesivas versiones, fijándose en 1978 en 30\% y en 1980 en 35\%. Desde 1996 se establece una bonificación diferenciada según el tamaño predial que oscila entre 30\% y $50 \%$.

\subsection{El contexto: paradigmas e instrumentos de la planificación funcionalista}

Para entender con mayor precisión el sentido y los posibles objetivos que esta normativa tuvo en el período analizado, es necesario relacionarla con paradigmas urbanísticos globales y con otros instrumentos y modalidades de planificación existentes en Chile en ese momento.

\subsubsection{El conjunto que se distingue de su contexto}

Así como el período 1930-1950 estuvo influido por Brunner, el período de la planificación funcionalista estuvo fuertemente marcado por el legado del CIAM y la visita de Le Corbusier a ciudades latinoamericanas como asesor de los nuevos organismos de planificación (Almandoz, 2007). Si bien en el Primer Congreso de Arquitectura y Urbanismo organizado por la Asociación de Arquitectos de Santiago en 1934 aún no se reconocen vínculos con los discursos del CIAM, ya a partir de 1935 se hace presente la influencia urbanística de Le Corbusier. En los seis números de la revista Arquitectura editados por Enrique Gebhardt, Waldo Parraguez y Mario Antonioletti en los años 1935-1936 se expresa la influencia de la visión funcionalista. Lo mismo ocurre en un escrito de Gebhardt publicado en Revista Zig-Zag con motivo del Primer Congreso Chileno de Urbanismo en 1937. Desde mediados de los años cuarenta, en artículos de la revista Arquitectura y Construcción se expresan ideas sobre la organización funcionalista de la ciudad planteada en la “Carta de Atenas" por el CIAM. Junto con la división de la ciudad en funciones, también llegaron las nuevas tipologías edificatorias modernistas. Para ejemplificar algunos alcances de esta influencia, están las imágenes del proyecto de título de Fernando Castillo Velasco, publicadas en la revista Arquitectura y Construcción. El trabajo se distingue por una marcada estética modernista de torres aisladas en un amplio parque (Castillo 1946, pp. 44-49). Castillo le asigna a esta nueva tipología de edificación aislada una connotación positiva de modernidad y caracteriza los "edificios que siguen la línea de fachada de tiempos coloniales" como algo ya obsoleto (Castillo 1946, p. 44).

Esta visión se recoge también en la propuesta morfológica para la "remodelación” de Santiago del Taller que Sergio Larraín dirigía en la PUC en 1946, evidentemente influenciada por el paradigma de la torre en el parque (Pérez de Arce 1946, pp. 30-69). El encargo de este taller es un "conjunto urbanístico" y en todos los trabajos de los alumnos el proyecto se superpone al contexto buscando la diferenciación en cuanto a volumetría, ubicación del edificio respecto de la línea de edificación y altura edificada. 


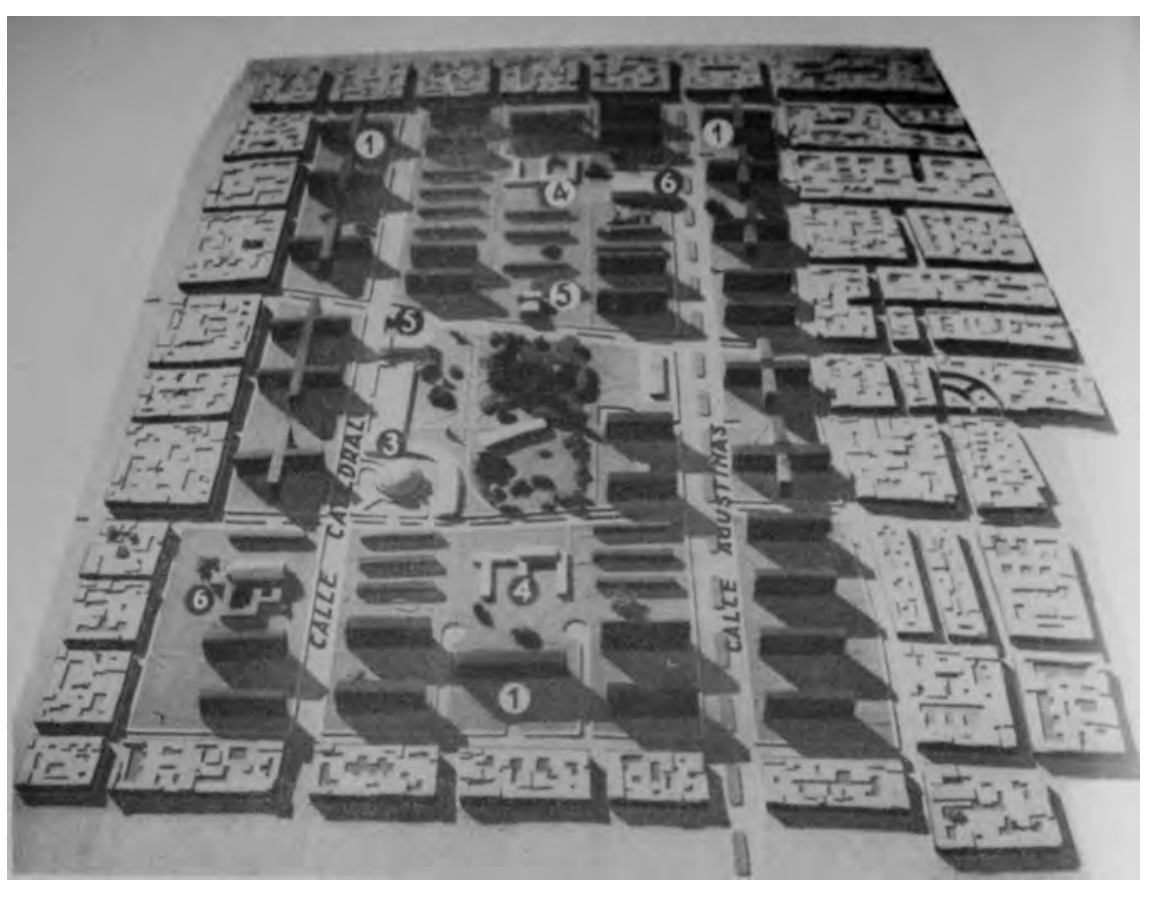

fuente Pérez de Arce, Mario (1946). Taller Sergio Larraín GM, Reestructuración de Cinco Sectores Residenciales de Santiago. ARQuitectura Y Constructión No 6, mayo 1946, r. 44. Santiago de Chile.

\subsubsection{El tamaño predial mayor}

Una de las principales motivaciones de la normativa de Conjunto Armónico en los años 60 es, según Amador Brieva, la necesidad de resolver el problema de la "tugurización" en la ciudad (Brieva, 2009). "Tugurización” es un término que aparece en nuestra literatura especializada desde los años treinta y se refiere a urbanizaciones carentes de higiene y espacio vital, como los "conventillos", y a la subdivisión irracional de la ciudad en lotes muy pequeños que albergan a gran cantidad de personas. Uno de los arquitectos que contribuyó a posicionar el tema del "tugurio" en el debate nacional fue Gebhardt, quien, siendo funcionario del Departamento de Estadísticas de la Municipalidad de Santiago, organizó en 1937 una exposición sobre el estado de los conventillos en Santiago (Exposición Ciudad y Vivienda) (Bedrak, 1986).

Fernando Castillo incorpora el concepto "tugurización" en su proyecto de título. Señala que se trata de una práctica negativa que es necesario revertir y argumenta a favor de una gestión más racional de la subdivisión predial, promoviendo mayores tamaños (Castillo 1946, p. 44).

En la misma línea, el mencionado Taller Larraín hace una propuesta funcionalista para la "remodelación" de Santiago (Pérez de Arce, 1946). Las condiciones generales impuestas a los alumnos muestran la envergadura con que se concebían 
los proyectos: un conjunto habitacional de dimensiones inusuales $\left(80.000 \mathrm{~m}^{2}\right.$ edificados) y áreas de intervención totales que oscilaban entre 22 y 78 hectáreas.

La reflexión académica y profesional en torno a la problemática de los "tugurios" y acerca del desarrollo de tamaños prediales mayores se cristaliza, finalmente, en el primer Plan Regulador Intercomunal de Santiago de 1960 (Honold y Poblete, 1966).

\subsubsection{La integración de usos complementarios a la vivienda y de espacios comu- nes abiertos}

El problema de los "tugurios" motivó la búsqueda de nuevas soluciones de vivienda a contar de los años cuarenta. En este contexto, se encuentran dos conceptos extranjeros que influenciaron la planificación y la legislación chilena en las décadas posteriores: el modelo de la "Unidad Vecinal" y el modelo funcionalista que integra vivienda con equipamiento.

No cabe duda que el medio fue influenciado por el concepto de las "Unidades Vecinales" (neighborhood units), que Clarence Perry desarrollara en 1920 para Nueva York (Lewis, 1929) ${ }^{33}$. Un artículo sobre la aplicación de este concepto en Lima, que data de 1945, fue publicado en la revista nacional Arquitectura y Construcción. En él se describe la falta de espacio interior y de esparcimiento de la vivienda tipo de un típico callejón (tugurio) limeño y se arguye que la posible solución es la "Unidad Vecinal" o, como la describen, una ciudad en miniatura "en la cual están resueltos los problemas de habitación, (...) de recreación, de comercio, para un grupo (...) de habitantes que (...) ascenderá a 5.000 por unidad ${ }^{34}$.

El Taller Larraín establecía que "las soluciones del conjunto urbanístico" debían "contemplar todas las facilidades fundamentales de la población: habitación, trabajo, recreación y circulación." Según se desprende de la descripción que hace Mario Pérez de Arce de este taller, el proyecto también incorporaba equipamiento en forma de escuelas, sectores comerciales, clubes sociales, teatros y áreas verdes. Para cumplir estos requerimientos el taller establecía explícitamente que se debían constituir los llamados neighborhood units, lo que muestra que ya en 1946, una década antes de su materialización, la academia santiaguina discutía la creación de las Unidades Vecinales.

Por otro lado, existen argumentos que muestran la influencia de la corriente modernista proclamada por el CIAM. Fernando Castillo establece que la ciudad debe ser pensada de manera colectiva y "armónica", más eficiente en términos de gestión, con economía de escala y con una distribución racional y generosa de espacios abiertos (ventilación y asoleamiento). A través de su crítica al desarrollo in-

33 El término neighborhood unit o unidad vecinal fue implementado en sus inicios por Clarence Perry para el Plan Regional de Nueva York, como una respuesta racional al crecimiento suburbano. Consistía en la versión norteamericana de la ciudad jardín, basada en el concepto de la unidad autosuficiente. La unidad vecinal de Perry se centraba en la escuela básica y el centro comunitario y estaba rodeada por arterias viales en las cuales se localizaban las viviendas multifamiliares, el comercio y los servicios. El tamaño ideal de la unidad era de 5.000 a 6.000 personas y estaba determinada por la población necesaria para constituir una escuela de 800 a 1.200 niños (el tamaño ideal para una escuela básica de acuerdo a los estándares de la época). Perry establecerá una serie de estándares en cuanto a porcentajes de áreas verdes, circulaciones y usos de suelo para distintos tipos de unidades vecinales.

34 Extracto de los artículos sobre tugurios o callejones y sobre las Unidades Vecinales publicados en junio y septiembre de 1945 en "El Arquitecto peruano" en: Arquitectura y Construcción (1946). 
mobiliario en el barrio residencial "Del Carmen" (sector Diagonal Paraguay), es posible conocer las lógicas del momento: racionalizar la infraestructura vial, reemplazando las "calles tortuosas" existentes por un sistema de calles de mayor tamaño que corten los paños de terreno en segmentos más grandes. En estos, Castillo dispone espaciosas áreas verdes y locales comerciales. Este último aspecto evidencia que los círculos académicos ya estaban trabajando sobre la base de los postulados del CIAM (Castillo, 1946). Desde los años cuarenta, las traducciones e interpretaciones de la Carta de Atenas son recurrentes en las revistas chilenas ${ }^{35}$.

FIGURA 8 | "Desorden plástico y ausencia de áreas libres”

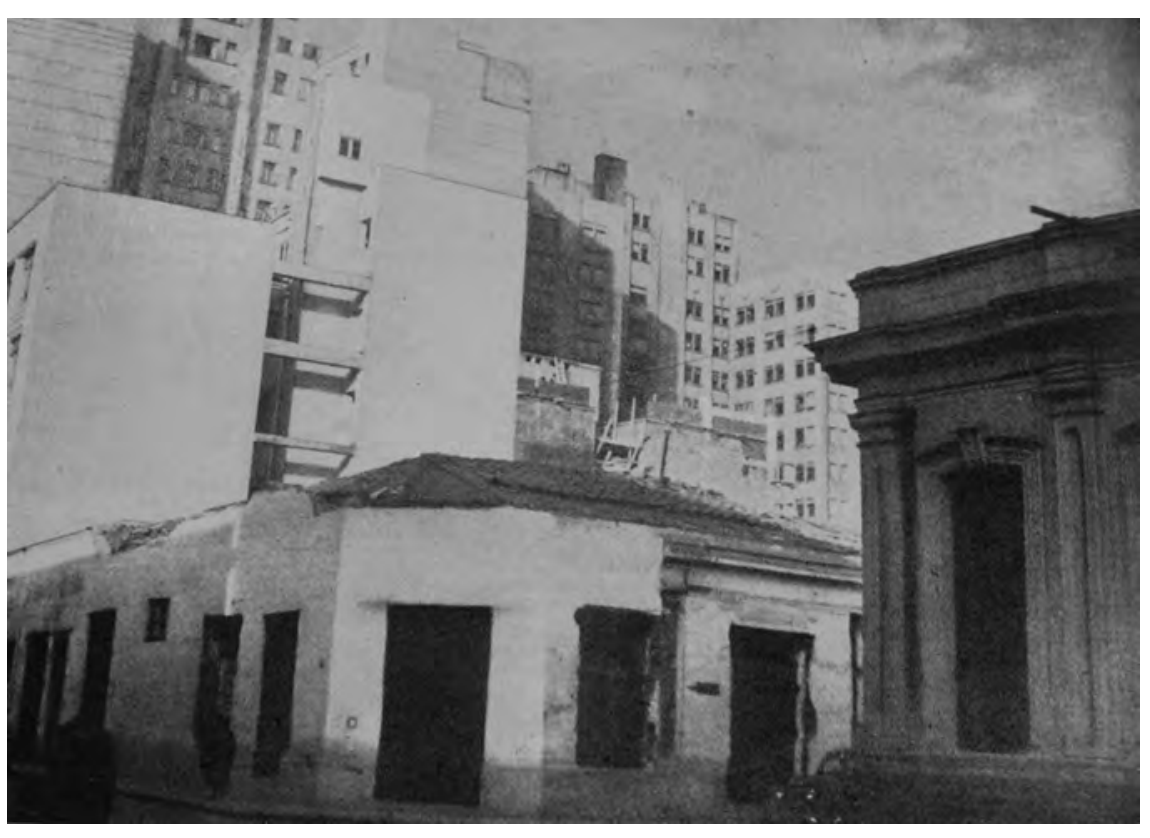

fuente Fotografía del artículo de Pérez de Arce sobre el Taller de Sergio Larraín 1946.

\subsubsection{Relación con "Zonas de Remodelación" del PRIS y las "Remodelaciones" de la CORMU}

Otra figura importante para entender el sentido que pudo tener la normativa de Conjunto Armónico en el Plan Regulador Intercomunal de Santiago de 1960 (PRIS) (36 $^{36}$ es la "Remodelación Urbana", gestionada por la CORMU (Corporación de Mejoramiento Urbano) entre 1965 y 1975 . La motivación para esto es comprobar la tesis planteada por Luis Eduardo Bresciani L., quien sostiene que el "Conjunto Armónico" constituye la versión normativa de proyectos de Remodelación Urbana que tradicionalmente habían sido llevados a cabo por la CORMU (Alcaíno, 2008, p. 263). 
La comprobación de dicha tesis pasa por conocer los instrumentos asociados a la "Remodelación". En primera instancia, cabe destacar que el PRIS de 1960 -elaborado por Juan Honold, Pastor Correa y Jorge Martínez, arquitectos de la Dirección de Planeamiento del MOP- se basó en los modelos funcionalistas propuestos por el CIAM y tuvo como referentes el "Greater London Master Plan" de Abercrombie y el modelo francés de reconstrucción de Postguerra elaborado por Gastón Bardet (Ponce de León 1996). En el PRIS de Santiago se hace referencia explícita a la planificación basada en la división funcional de la ciudad en zonas para habitación, trabajo, circulación y esparcimiento (Honold 1957, 1966). Asimismo, el PRIS establece la posibilidad de destinar zonas de "Remodelación Urbana" en las comunas.

Creada en 1965, la CORMU fue la institución destinada a llevar a cabo proyectos de "Remodelación" como la "Unidad Vecinal Portales" o la "Remodelación San Borja”. Su objetivo fue promover la remodelación urbana ${ }^{37}$ en una modalidad de gestión mixta, público-privada (Raposo, 2004). La CORMU tuvo atribuciones para determinar zonas de remodelación, comprar y expropiar terrenos y compensar e indemnizar la venta de terrenos (CChC, 1964). Un estudio de la Cámara Chilena de la Construcción permite aseverar que existe un vínculo directo entre la institucionalidad de la CORMU y la norma del Conjunto Armónico de 1963, cuyo sentido sería el de fijar el marco legal para gestiones CORMU (CChC, 1964).

La estrecha relación entre el Conjunto Armónico y la figura de la "Remodelación” en el marco de la CORMU se comprueba también al comparar los ámbitos regulados y los indicadores (estándares) utilizados para especificar la regulación. A continuación se compara un ejemplo paradigmático de los proyectos CORMU realizados entre 1966 y 1976, la "Remodelación San Borja"38, con la reglamentación del Conjunto Armónico de la OGUC de $1970^{39}$.

CUADRO 1 | Tabla comparativa de indicadores entre disposiciones normativas en la OGUC y cifras resultantes en el proyecto de Remodelación San Borja

\begin{tabular}{l|l|l}
\hline & \multicolumn{1}{|c|}{ OGUC $1970 / 1978$} & \multicolumn{1}{c}{ CORMU SAN BORJA } \\
\hline $\begin{array}{l}\text { DifERENCIACIÓN DEL ENTORNo } \\
\text { SUPERFICIE DEL TERRENO }\end{array}$ & “distinto del carácter general del barrio" & $\begin{array}{l}\text { Diferenciación tipológica: } \\
\text { torre aislada v/s manzana compacta. }\end{array}$ \\
$\begin{array}{l}\text { EXCEPCIÓN A LA NORMATIVA } \\
\text { VIGENTE }\end{array}$ & $\begin{array}{l}50 \% \text { más de densidad o 30\% más de } \\
\text { constructibilidad }\end{array}$ & $\begin{array}{l}\text { Aproximadamente } 182.000 \mathrm{~m}^{2} \\
1.042 \text { habitantes/hectárea } \\
\text { Coeficiente de constructibilidad } 1,7\end{array}$ \\
PROGRAMA & $\begin{array}{l}\text { Equipamiento comunitario: mercado, } \\
\text { gimnasio, templo, teatro, hospital, etc. }\end{array}$ & Comercio, escuela, guardería, museo. \\
ÁREA COMÚN LIBRE & $6 \mathrm{~m}^{2} /$ habitante & $6,63 \mathrm{~m}^{2} /$ habitante \\
\hline
\end{tabular}

FUENTE SCHLACK, 2010.

37 Ley No 16.391 D.O. 16.12.1965, Artículo 43.

38 Superficie calculada a partir de información publicada sobre la Remodelación San Borja en Revista AUCA No 16 agosto-septiembre 1969.

39 Art. 480-486 de la OGUC, DS No 107 MINVU DO 5.6.1970. 


\subsection{Plaza Lyon: un caso de la normativa de "Conjunto Armónico"}

FIGURA 9 | Vista aérea del Conjunto Plaza Lyon y Centro Nuevo, 1980

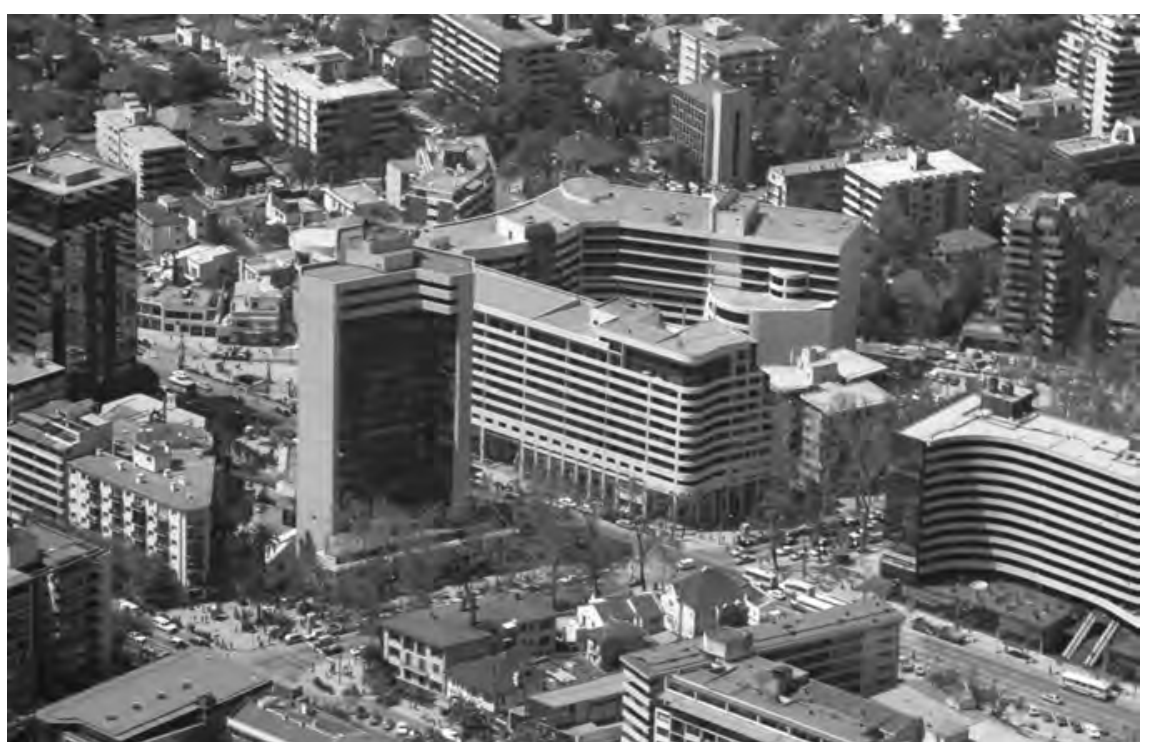

fuente Archivo personal Pedro Murtinho.

En 1975 desaparece la CORMU y la normativa de Conjunto Armónico entra en una nueva fase. A contar de entonces, constituirá el marco legal para la puesta en práctica de proyectos de relevancia urbana e iniciativa privada que antes habían sido posibles en la institucionalidad definida por la CORMU.

El conjunto Plaza Lyon ejemplifica de manera apropiada la normativa de Conjunto Armónico en sus versiones de 1970 y 1976. Este período se caracteriza por seguir el espíritu iniciado por los proyectos de remodelación: tamaños prediales mayores, aumento sustancial en la constructibilidad, diferenciación tipológica respecto del entorno, incorporación de equipamiento comunal y espacios libres comunes. Sin embargo, a diferencia de las Unidades Vecinales y los grandes proyectos de remodelación de los años sesenta, el conjunto Plaza Lyon se concibe en el marco de paradigmas postmodernistas. Predominan las tipologías edificatorias que reinterpretan las tipologías históricas de Santiago, destacando la morfología de manzana compacta e interior con galerías. Tal como señala el arquitecto Pedro Murtinho, quien proyecta Plaza Lyon junto a su oficina a fines de los años setenta, el conjunto considera el uso de tipologías históricas, tales como el portal, la manzana, el block, la plaza, la recreación de la fachada interior y la fachada continua (Fernández, 1991, pp. 10-15) ${ }^{40}$.

Murtinho, P. (Mayo 2010), Entrevista realizada por Elke Schlack. 
En la concepción del proyecto, los autores siguen la aproximación de Christopher Alexander y los arquitectos postmodernos Léon Krier y James Stirling. De esta manera, el proyecto aborda simultáneamente la construcción del edificio y su dimensión urbana (Fernández, 1991, pp. 10-15). La condición urbana que busca el proyecto radica en lo que Teodoro Fernández define como "una nostalgia por el orden perdido de la ciudad republicana, nostalgia de la riqueza social y de usos”. Así, este proyecto articula la división de la ciudad en suelo público y privado, haciendo uso de elementos como el portal y la plaza para resolver los espacios urbanos y transformar el edificio en un "monumento capaz de caracterizar un trozo de ciudad."

Sin embargo, el proyecto Plaza Lyon también se hace cargo de la demanda funcionalista por generar edificios complejos y de múltiples actividades. El edificio se compone de usos habitacionales, comerciales (supermercado y tiendas), oficinas y estacionamientos. Asimismo, se incorpora de manera orgánica a la planificación de la "Nueva Providencia" y al trazado de la Línea 1 del Metro, conectándose a la mesanina de la estación de metro Los Leones.

FIgURa 10 | Plano de la resolución de Conjunto Armónico Nr. 30 del 31/10/1977

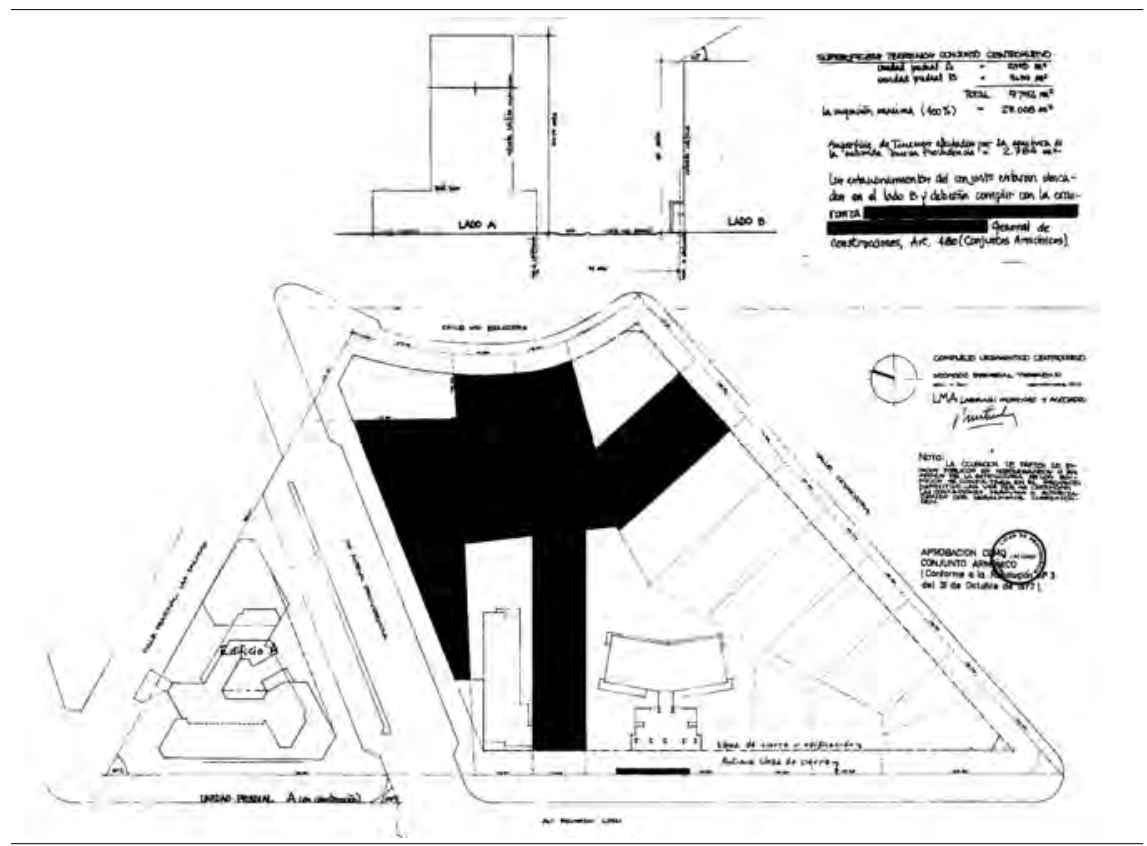

fuente Documentos de Catastro Ilustre Municipalidad de Providencia.

El permiso de edificación, otorgado en 1981, define que este edificio estaría acogido a la excepción normativa de Conjunto Armónico ${ }^{41}$. Como se observa a continuación, este proyecto establece estándares similares a los que presenta la Re-

41 Permiso de Edificación No. 92 de 1981. Resolución de Conjunto Armónico No. 16 del 26.12.1980. Documentos y planimetrías del expediente municipal. Departamento Catastro Ilustre Municipalidad de Providencia. 
modelación San Borja, de acuerdo a lo que establecía la normativa de Conjunto Armónico vigente en su época de construcción.

Este ejemplo de conjunto armónico presenta una gestión privada que articuló los intereses de al menos 17 propietarios involucrados ${ }^{42}$. La lógica subyacente al modelo de gestión fue la "unidad predial", que pretendía organizar la agrupación de predios en unidades urbanas como la manzana, en función de promover una renovación urbana acorde a las unidades morfológicas de la ciudad y no en función de la a veces arbitraria fusión espontánea que surge de la especulación inmobiliaria. El municipio de Providencia promovía en ese entonces la transformación del área central de la comuna de acuerdo a "unidades prediales" que constituyen, a la vez, una unidad de gestión inmobiliaria y una unidad morfológica como la manzana ${ }^{43}$.

Esta modalidad permitió el desarrollo coordinado del trazado de la Nueva Providencia, al mismo tiempo que la cualificación morfológica de las esquinas de esta nueva calle con la avenida Ricardo Lyon, en ese momento cruce significativo de la comuna de Providencia y la ciudad de Santiago.

CUADro 2 | Tabla comparativa de indicadores de la normativa de Conjunto Armónico de 1970 y Conjunto Plaza Lyon

\begin{tabular}{|c|c|c|}
\hline ASPECTO REGULADO & OGUC 1970 / 1978 & Conjunto Plaza Lyon \\
\hline UNIDADES PREDIALES MAYORES & $>2.500 \mathrm{~m}^{2}$ ó $33 \%$ manzana & $7.054 \mathrm{~m}^{2}$ \\
\hline Programas mixtos & Equipamiento comunitario. & $\begin{array}{c}\text { Vivienda, locales comerciales, } \\
\text { estacionamientos, oficinas, } \\
\text { supermercado }\end{array}$ \\
\hline ÁREA COMÚN LIBRE & $6 \mathrm{~m}^{2} /$ habitante & $\begin{array}{c}5 \mathrm{~m}^{2} / \text { habitante en espacios } \\
\text { comunes }\end{array}$ \\
\hline DENSIDAD HABITACIONAL & $50 \%$ más de densidad & 2.671 habitantes/ hectárea \\
\hline $\begin{array}{l}\text { COEFICIENTE DE } \\
\text { CONSTRUCTIBILIDAD }\end{array}$ & $30 \%$ más de constructibilidad & $\begin{array}{c}\text { Máxima admitida: } 8.45 \text { y } \\
\text { realizada: } 6.6\end{array}$ \\
\hline DIFERENCIACIÓN DEL CONTEXTO & Densidad y tipología edificatoria & $\begin{array}{l}\text { Difiere de la tipología "Placa- } \\
\text { Torre" prevista para esa zona, } \\
\text { propone edificación continua. }\end{array}$ \\
\hline
\end{tabular}

fuentes Revista AUCA No 16, Agosto/Septiembre 1969 y Art. 480 OGUC 1970, DS No 107 MINVU DO 5.6.1970 (SCHLACK, 2010).

42 Murtinho, P. (2010). Entrevista realizada por Elke Schlack en , mayo 2010.

43 Entrevistas realizadas a Germán Bannen y Rodrigo Rojas por Elke Schlack en mayo 2010. 
FIGURA 11 | Comparación de las áreas comunes libres disponibles en el caso del Conjunto Plaza Lyon (izquierda) y la Remodelación San Borja (a la derecha)

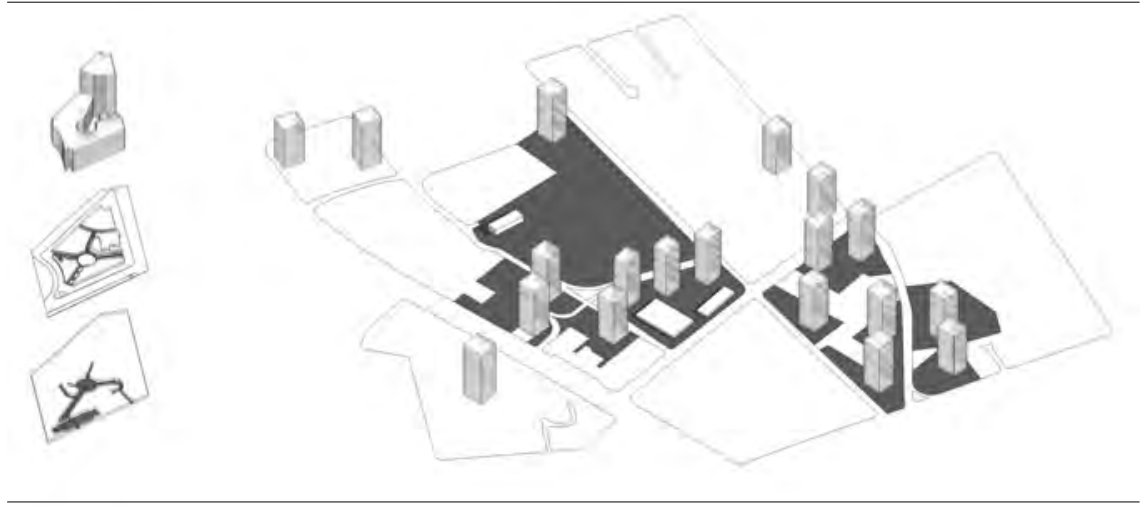

FUENTE ELABORACIÓN PROPIA, 2010.

\section{Conclusión}

La norma de Conjunto Armónico está incidiendo fuertemente en la nueva morfología de Santiago.

Concebida para regular el diseño urbano, los objetivos originales de la norma de Conjunto Armónico están aún presentes en la normativa actual. Sin embargo, hay muy pocos casos que persiguen hoy esos objetivos iniciales.

Un ámbito de dichos objetivos es la regulación de una armoniosa interacción con el patrimonio preexistente, un recurso muy poco usado en las últimas décadas, en las cuales la investigación identificó sólo dos casos: el "Hotel Plaza San Francisco" ${ }^{44}$ y la "Congregación de la Providencia” ${ }^{45}$. En la actualidad, la mayoría de los casos de "conjuntos armónicos" situados en un entorno patrimonial se acogen a otro artículo que permite acentuar la edificabilidad, sin importar el contexto. Un caso paradigmático es el conjunto armónico "Bellavista Forestal" ${ }^{46}$, consistente en tres torres aisladas de 19 pisos cada una (aproximadamente 60 metros) que se inserta en un barrio de fachada continua y alturas que fluctúan entre los cuatro y doce metros. Así, resulta particularmente paradójico el nombre de dichos conjuntos, cuando a pesar de la declaratoria patrimonial de la comuna, es otro artículo de la misma normativa de Conjunto Armónico vigente el que permite transgredir dichas declaratorias patrimoniales ${ }^{47}$.

Un segundo aspecto es la regulación de unidades arquitectónicas que aporten al diseño urbano. Con esta finalidad, se definía el envolvente máximo a través de la

44 Edificio ubicado en la vereda sur de la Avda. Alameda esquina calle Londres, junto al monumento nacional Iglesia San Francisco, construido en la década de los 80.

45 Conjunto armónico consistente en un monumento nacional ubicado en la vereda sur de la Avda. Providencia entre las avenidas Condell y Salvador, declarado Conjunto Armónico en la década de los 70.

46 Conjunto ubicado en la intersección de calle Bellavista y Avda. Pío Nono.

47 Al respecto ver Plan Regulador Comunal de Recoleta (2009), Plano 4 “Patrimonio”, ICH-8 Plan Regulador Comunal de Recoleta. Y revisar material en archivo de "Ciudad Viva”, organización ciudadana. 
línea de edificación continua y la altura de edificación. Al reemplazar la forma de fijar la volumetría por los indicadores "rasante" y "coeficiente de constructibilidad", se produce un drástico cambio en la formulación de la norma. Desde entonces, en predios de una misma manzana, la morfología resulta totalmente distinta dependiendo de los tamaños prediales (Figuras 12 y 13). Tal como se observa en la tabla (Figura 16), desde los años noventa en los conjuntos armónicos coincide el máximo volumen construible condicionado por estas normas con la volumetría final del edificio. Consecuentemente, la morfología edificatoria es definida por la máxima constructibilidad, a la cual se supeditan las otras condiciones de edificación, como la ocupación de suelo o el distanciamiento a medianeros. Tal como se refleja en los

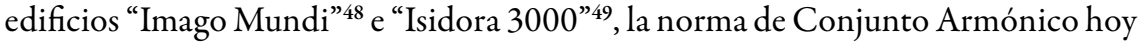
no necesariamente constituye un instrumento que permita controlar la morfología de un grupo de edificaciones. Entonces, cabe preguntarse si el Conjunto Armónico, al aplicarse sobre edificaciones que constituyen importantes piezas urbanas, no debiera estar regulado también por normas de morfología urbana.

FIGURA 12 Definición esquemática de la volumetría a partir de una rasante de $70^{\circ}$ en predios con frente predial de 25 metros (A), 50 metros (B) y 15 metros (C). La morfología de las edificaciones queda definida por el tamaño predial

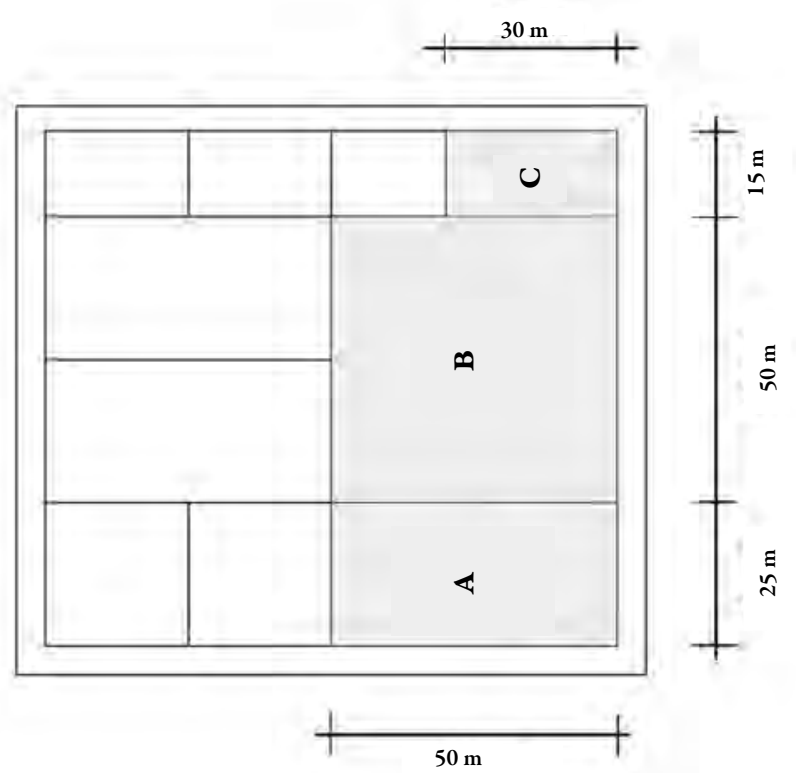

FUENTE ELABORACIÓN PROPIA 2010. 
FIGURA 13 | Definición esquemática de la volumetría a partir de una altura y agrupamiento fijo para los predios $\mathrm{A}, \mathrm{B}$ y $\mathrm{C}$

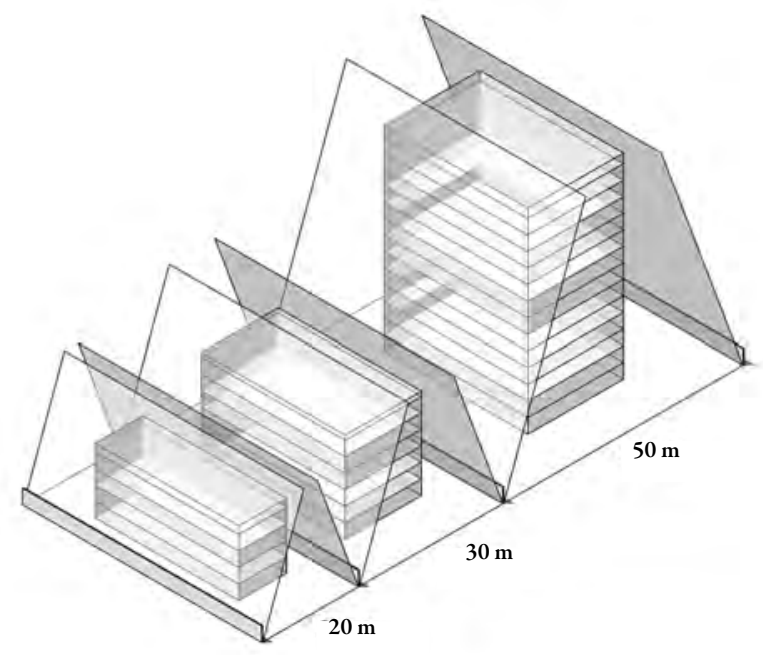

FUENTE ELABORACIÓN PROPIA 2010.

Un tercer ámbito en que se refleja la pérdida de los objetivos iniciales es la forma en que se gestionan las excepciones. Si bien en su origen las exigencias de altura, ventilación y asoleamiento estaban menos sistematizadas que hoy, el Director de Obras representaba la figura de garante del cumplimiento de aquellos valores de las edificaciones. Incluso, en caso de divergencia entre el director de obras y el arquitecto proyectista, existía una figura de arbitraje, representada por tres arquitectos. Actualmente, la coherencia del proyecto en los requerimientos morfológicos y funcionales está fuera del control del Municipio y los planificadores locales.

Un cuarto aspecto en que esta normativa se ha alejado de su sentido original es el "proyecto integral", el cual incluye en el proyecto no sólo el edificio, sino también áreas verdes, equipamiento y vialidad. Los proyectos acogidos a dicha norma en la década de los 80, tales como el edificio "Plaza Lyon", el edificio “Santiago Centro", la “Torre Santa María” y el edificio "Forum” proveían de un generoso "espacio común libre" ( $6 \mathrm{~m}^{2}$ por habitante), cesión que hoy se encuentra sustituida por espacios individuales en forma de terrazas o balcones. Ejemplos de esto son el edificio "Imago Mundi", el edificio "Isidora 3000", el edificio "Edificio Arrau"50 y el edificio "Bellavista Forestal", cuyos espacios comunes libres fluctúan entre 1 y $3 \mathrm{~m}^{2}$ por habitante (Cuadro 3). Cuando se elimina la obligación de proveer espacio común en la norma, tácitamente se derogó la responsabilidad del gestor en estas materias. Cabe preguntarse quién debiera hacerse cargo de esta responsabilidad, sobre todo considerando la escala de proyecto urbano que estos proyectos comúnmente tienen. 
Tal como sucede con las exigencias de área verde, la normativa referente al "equipamiento comunitario" también sufre una reducción. Inicialmente, la normativa promovía la creación de mercados, gimnasios, templos, teatros y hospitales; en la actualidad son contados los casos que incorporan equipamientos comunales, siendo los usos predominantes los de vivienda y oficinas. Sólo el edificio "Imago Mundi" incorpora en su conjunto un jardín infantil y una biblioteca. Aún no queda claro qué relevancia tendrían la mayoría de los actuales "conjuntos armónicos" en definir subcentros (Cuadro 3).

Un quinto ámbito en que la norma se ha modificado se refiere al desarrollo de paños prediales mayores como forma más "armoniosa" de desarrollar la ciudad. La condición para acogerse a la norma de Conjunto Armónico ha sido y es, hasta hoy, contar con un predio mayor a $2.500 \mathrm{~m}^{2}$. La forma en que se premia el uso de predios mayores es el aumento del volumen construido. Sin embargo, se produce el siguiente contrasentido: al favorecer una excesiva densidad sin controlar la morfología, se corre el riesgo de un desarrollo arbitrario, esta vez no para evitar el "tugurio" sino para promover la construcción de "piezas urbanas". En este contexto, el edificio Arrau y el Plan Maestro asociado constituyen un ejemplo a seguir. Falta debatir acerca de cuáles serán los mecanismos que integren la planificación comunal con los conjuntos armónicos, en el sentido de lo que propone la actual modificación de la Ley: otorgar a los municipios la atribución de decidir las zonas en que se pueden localizar estos conjuntos.

Un sexto aspecto, que se refiere a permitir tipologías divergentes respecto del entorno, se remonta a un momento en que diferenciarse del entorno era plenamente aceptado y generalmente se expresaba en el reemplazo del paradigma de la ciudad continua por la ciudad dispersa. Sin embargo, como el debate actual demuestra que existe una sensibilidad mayor en el manejo de lo preexistente, parece necesario repensar esa relación. Los casos actuales de "conjuntos armónicos" no sólo no son explícitos en su concepción tipológica, sino que más bien se presentan como tipologías híbridas. El "Imago Mundi” es una especie de torre compacta y las torres de "Bellavista-Forestal" corresponden a una torre aislada, que en algunos puntos adopta arbitrariamente la línea oficial (Figura 15).

Del anterior análisis se puede concluir que, si bien el Conjunto Armónico constituye un incentivo interesante para la renovación urbana, es necesario precisarlo como una herramienta para potenciar grandes proyectos de renovación urbana con un rol más estratégico dentro de los barrios donde se insertan.

Concebida originalmente para regular la creación de edificios excepcionales en la ciudad, en la actualidad se ha transformado en una fórmula para lograr mayor constructibilidad, sin que necesariamente un mayor volumen edificado implique que se constituya en un edificio de mayor relevancia en la ciudad. 


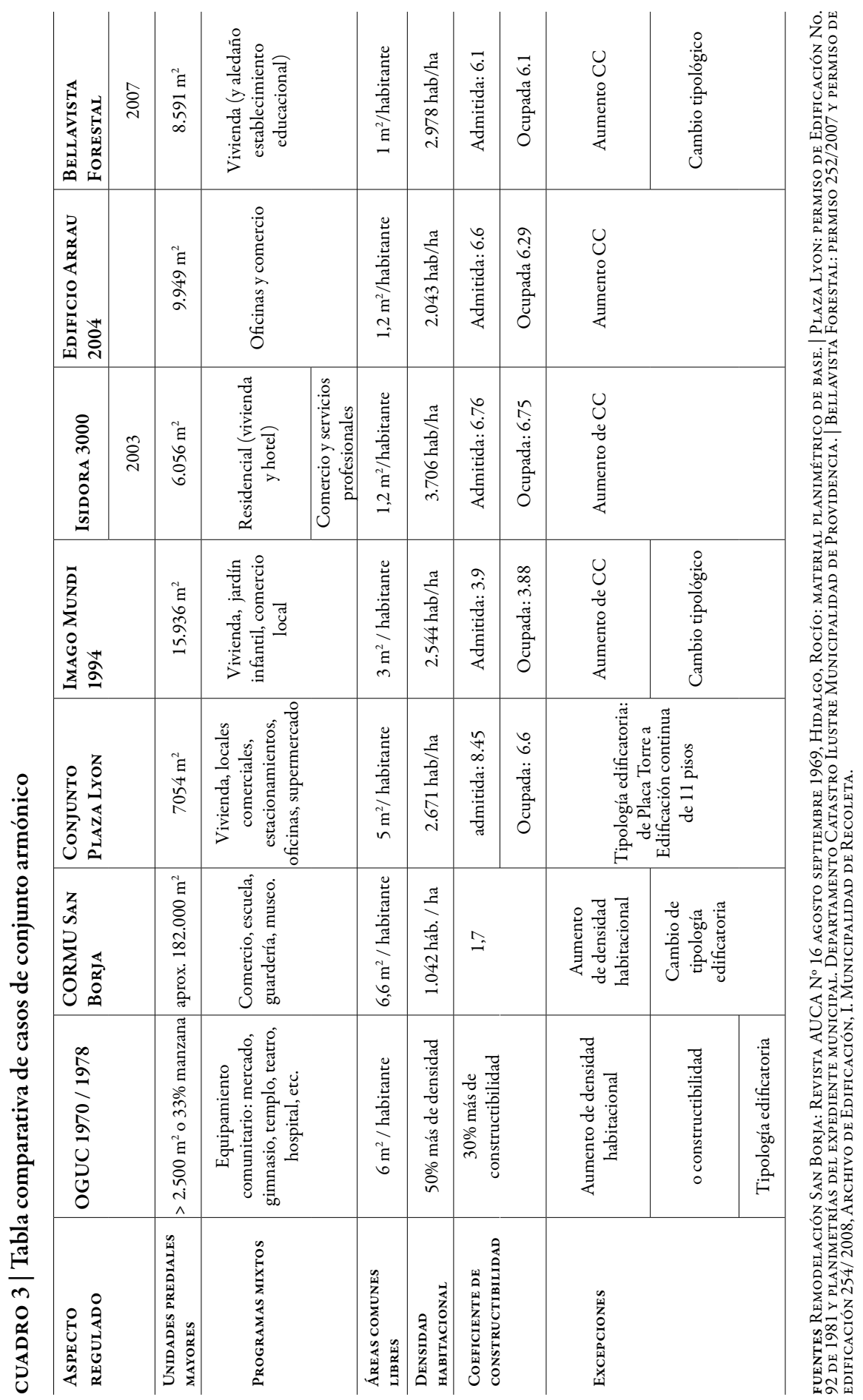


FIGURA 14 | Comparación de casos y sus constructibilidades

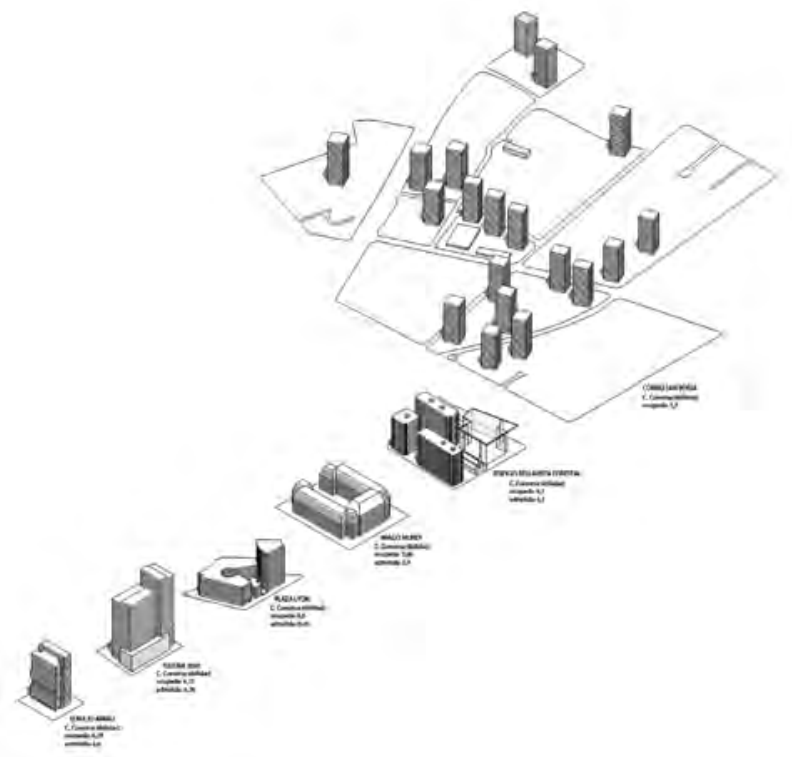

FUENTE SCHLACK, 2010.

FIGURA 15 | Comparación de la morfología del conjunto armónico y la morfología prevista en el Plan Regulador Comunal, para el Conjunto Plaza Lyon (izquerda) y el Conjunto Bellavista Forestal (derecha)
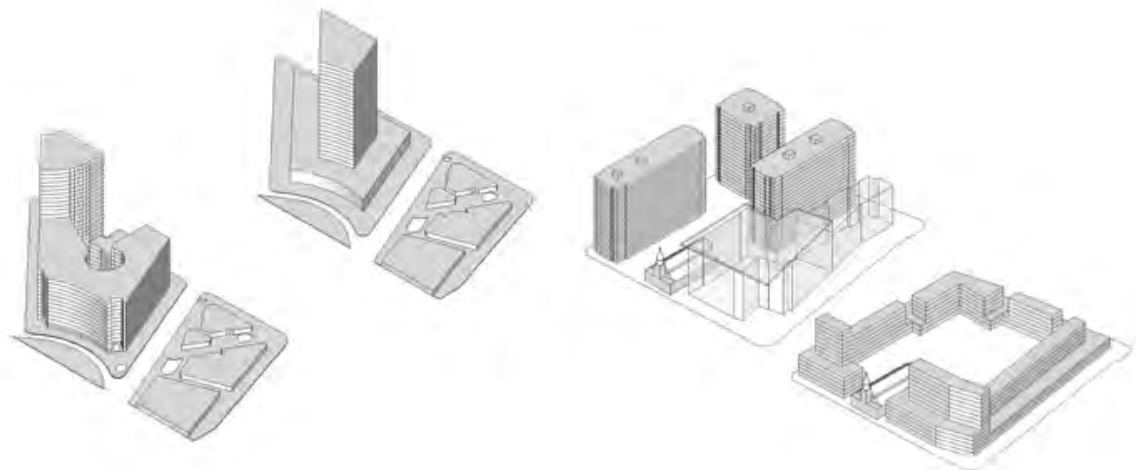

FUENTE ELABORACIÓN PROPIA. 


\section{Referencias bibliográficas}

Aguirre, B. (2005). Espacio Público y Arte Cívico en Santiago, 1930-1950. Una lectura a través de proyectos singulares. Recuperado el 10 de junio de 2010, del sitio web de la Universidad Central: http://www.ucentral.cl/fid/pdf/i_ciudad_sociedad/espacio_publico_arte_civico.pdf

Alcaíno, J. (2008). Efectos de las normas de excepción sobre la planificación urbana comunal y sobre los conflictos urbanos. Santiago de Chile: Tesis de Magíster del Instituto de Estudios Urbanos, Facultad de Arquitectura, Diseño y Estudios Urbanos Universidad Católica de Chile.

Almandoz, A. (2007). Modernización urbanística en América Latina. Luminarias extranjeras, cambios disciplinares, 1900-1960. Revista Iberoamericana, Vol. VII, No. 27, pp. 59-78.

Bedrak, M. (1986). El Urbanismo en Chile 1928-1973. Revista C.A. No. 46, pp. 31-32. Santiago de Chile: Editorial Revista C.A.

Brieva, A. (2009). Entrevista a Amador Brieva, realizada por las autoras del artículo en septiembre 2009.

Brunner, K. (1996). Primera Conferencia en la Universidad de Chile. Revista de Arquitectura No. 8, Julio-Diciembre. Santiago de Chile: Facultad de Arquitectura y Urbanismo, Universidad de Chile.

Brunner, K. (1932). Santiago de Chile. Su estado actual y futura formación. Santiago de Chile: Imprenta La Tracción.

Brunner, K. (1939). Manual de Urbanismo. Bogotá: Concejo Municipal de Bogotá, Imprenta Municipal.

Brunner, K. (1930). Problemas actuales de urbanización. Anales de la Universidad de Chile, 2a Serie, Primer Trimestre, año VIII. Santiago de Chile: Universidad de Chile.

Brunner, K. (1930). Seminario de Urbanismo. Revista Comuna y Hogar N 13. Santiago de Chile.

Cáceres, G. \& Sabatini, F. (2001). Santiago de Chile: la recuperación de plusvalias puesta en perspectiva histórica. En M. Smolka y F. Furtado (Ed.). Recuperación de Plusvalías en América Latina. Santiago de Chile: Eurelibros. Instituto de Estudios Urbanos y Lincoln Institute.

Cámara Chilena de la Construcción (1964). Estudio sobre Corporación de Mejoramiento Urbano. Revista de la Construcción CChC Noviembre, pp. 63-69. Santiago de Chile.

Castillo, F. (1946). El Barrio Residencial El Carmen. Revista Arquitectura y Construcción No. 2, p. 43, Enero. Santiago de Chile.

Fernández, T. (1991). La oficina Murtinho \& Asociados Arquitectos. Santiago de Chile: Editorial ARQ.

Gurovich, A. (1996). La venida de Karl Brunner en gloria y majestad. Revista de Urbanismo No. 8. Santiago de Chile: Facultad de Arquitectura y Urbanismo, Universidad de Chile.

Hofer, A. (2003). Karl Brunner y El Urbanismo Europeo en América Latina. Bogotá: El Áncora Editores - Corporación de la Candelaria.

Honold, J. (1957). Visión General de los problemas del Gran Santiago. Discurso en el Seminario del Gran Santiago 1957. Revista de Urbanismo, Universidad de Chile, Santiago de Chile.

Honold, J. \& Poblete, J. (1966). Radiografía de la Metrópoli. El Plan Regulador Intercomunal de Santiago. $A U C A$, Vol. 1, No. 2. Santiago de Chile.

Pérez, F. (1991). Le Corbusier y Sudamérica, viajes y proyectos. Santiago de Chile: Escuela de Arquitectura, Pontificia Universidad Católica de Chile. 
Pérez, F. \& Rosas, J. (2002). Cities within the city: Urban and Architectural transfers in Santiago de Chile, 1840-1940. Londres: Routledge. Taylor and Francis Group.

Pérez de Arce, M. (1946). Taller Sergio Larraín GM, Reestructuración de Cinco Sectores Residenciales de Santiago. Arquitectura y Construcción, No. 6, Mayo, Santiago de Chile.

Perry, C. (1929). The Neighborhood Unit. En H.M. Lewis (Ed.). Regional Plan for New York and its Environs, Neighborhood and Community Planning Vol. 7. New York.

Peterson, J. A. (2002). The City Beautiful Movement: Forgotten Origins and Lost Meanings. En D. A. Krueckeberg (Ed.). Introduction to Planning History in the United States. New Jersey: Center for Urban Policy Research, Rutgers, State University of New Jersey.

Ponce de León, M. (1996). Intercomunal de Santiago. Plan Regulador MOPT 1960. Serie Nacional de Urbanismo No. 1, pp. 40-47.

Revista Zig-Zag (1937). Primer Congreso Chileno de Urbanismo. Diciembre, Santiago de Chile.

Raposo, A. \& Valencia, M. (2004). Modernidad, Diseño Urbano y Utopía: Notas sobre el fundamento político de las acciones de Remodelación Urbana en Santiago. El caso de CORMU 1966 1973. Revista de Urbanismo No. 9. Santiago de Chile: Facultad de Arquitectura y Urbanismo, Universidad de Chile.

Rosas, J. (2009). La Vivienda Moderna en el Centro de Santiago. En A. Téllez (Ed.). Vivienda Multifamiliar en Santiago, 1930-1970. Santiago de Chile: Salviat Impresores. Pontificia Universidad Católica de Chile y Universidad Diego Portales.

Sitte, C. (1965). City planning according to artistic principles. New York: Random.

Revista Arquitectura y Construcción (1945). Avenida General Manuel Bulnes. Revista Arquitectura y Construcción, No. 1. 


\section{Anexo 1}

\section{Norma de conjunto armónico presente en la Ordenanza General de Urbanismo y Construcciones}

Artículo 2.6.4. Para los efectos previstos en los artículos 107, 108 y 109 de la Ley General de Urbanismo y Construcciones, se considerará que un proyecto tiene la calidad de conjunto armónico, cuando cumple con alguna de las condiciones que se señalan a continuación y con las exigencias que para cada caso se establecen, sin perjuicio de lo prescrito en los artículos 2.6.15. y 2.6.16. de este mismo Capítulo 2.

1 Condición de dimensión:

a Estar emplazado en un terreno cuya superficie total sea igual o superior a 5 veces la superficie predial mínima establecida por el Plan Regulador respectivo, siempre que la superficie total no sea inferior a $5.000 \mathrm{~m}^{2}$;

b Estar emplazado en un terreno que constituya en sí una manzana existente, resultante o no de una fusión predial, cualquiera sea la superficie total de ésta;

c Estar emplazado en un terreno resultante de una fusión predial, conforme al artículo 63 del D.F.L. No 458, de Vivienda y Urbanismo, de 1975, siempre que su superficie no sea inferior a $2.500 \mathrm{~m}^{2}$.

2 Condición de uso:

Estar destinado a equipamiento y emplazado en un terreno en que el Plan Regulador respectivo consulte el equipamiento como uso de suelo, que tenga una superficie total no inferior a $2.500 \mathrm{~m}^{2}$ y cumpla con lo preceptuado en el artículo 2.1.36. de esta Ordenanza.

El distanciamiento entre los edificios que contempla el proyecto y los deslindes con los predios vecinos, no podrá ser inferior a $10 \mathrm{~m}$., aunque se emplace en áreas de construcción continua obligatoria.

3 Condición de localización y ampliación:

a Estar localizados en el mismo terreno en que estén emplazados inmuebles declarados Monumentos Nacionales o definidos por el Plan Regulador como inmuebles de conservación histórica y que el proyecto contemple su ampliación, restauración, remodelación, reparación o rehabilitación. En el primer caso deberán contar con autorización previa del Consejo de Monumentos Nacionales, conforme a la Ley No 17.288 y en el segundo, con autorización de la Secretaría Regional Ministerial de Vivienda y Urbanismo.

b Estar emplazados en un predio contiguo a un inmueble declarado Monumento Nacional o definido por el Plan Regulador como inmueble de conservación histórica y que el proyecto contemple la ampliación, restauración, 
remodelación, reparación o rehabilitación del respectivo Monumento Nacional o inmueble de conservación histórica.

En estos casos, la ampliación, restauración, remodelación, reparación o rehabilitación del inmueble declarado Monumento Nacional deberá contar con autorización previa del Consejo de Monumentos Nacionales, conforme a la Ley No 17.288 , y cuando dichas obras se realicen en un inmueble de conservación histórica, con la autorización previa de la Secretaría Regional Ministerial de Vivienda y Urbanismo.

Los proyectos que cumplan con alguna de las condiciones señaladas en el inciso anterior deberán, además, cumplir copulativamente con los siguientes requisitos:

a Que el terreno enfrente en al menos 20 metros a una vía existente o proyectada en el Plan Regulador respectivo, de un ancho mínimo de $20 \mathrm{~m}$, con calzada de no menos de $14 \mathrm{~m}$.

En el caso de que la calzada no se encuentre materializada con las características señaladas, deberá ser ensanchada por el proyecto, hasta encontrarse o empalmarse con una calzada existente de al menos el mismo ancho.

b Que se ejecute un proyecto de mejoramiento en el espacio público que enfrenta el terreno, en toda el área comprendida entre la línea oficial y la solera, de acuerdo al diseño y características establecidos en el respectivo plano seccional.

Sólo se podrán hacer las exigencias a que alude la letra b) del inciso anterior, cuando las Municipalidades hayan establecido las características y el diseño de los proyectos de mejoramiento del espacio público mediante planos seccionales.

Las obras que se deban realizar en conformidad al presente artículo deberán ejecutarse o garantizarse y contar con recepción final previa o conjuntamente con las obras de edificación.

4 Artículo 2.6.5. Los proyectos que cumplan con la condición de dimensión a que se refieren las letras a) o b) del número 1. del artículo 2.6.4, podrán exceder hasta en un 50\% el coeficiente de constructibilidad establecido por el Plan Regulador respectivo.

5 Los proyectos que cumplan con la condición de dimensión a que se refiere la letra c) del número 1. del artículo 2.6.4, podrán exceder hasta en un $30 \%$ el coeficiente de constructibilidad establecido por el Plan Regulador respectivo.

Artículo 2.6.6. Los proyectos que cumplan con la condición de uso podrán exceder hasta en un $50 \%$ el coeficiente de constructibilidad establecido por el Plan Regulador respectivo.

Artículo 2.6.7. Los proyectos que cumplan con la condición de localización y ampliación a que se refieren las letras a) o b) del numeral 3. del artículo 2.6.4. de esta Ordenanza, podrán aumentar hasta en un $50 \%$ el coeficiente de constructibilidad establecido en el Plan Regulador respectivo. 
Artículo 2.6.8. Tratándose de fusión de terrenos, en los porcentajes de incremento de los coeficientes de constructibilidad señalados en los artículos 2.6.5., 2.6.6. y 2.6.7. se entiende incluido el $30 \%$ de aumento de dicho coeficiente que otorga el artículo 63 del D.F.L. No 458, de Vivienda y Urbanismo, de 1975.

Artículo 2.6.9. Los proyectos que cumplan con la condición de dimensión y/o de uso, podrán exceder hasta en un $25 \%$ la altura máxima establecida por el Plan Regulador respectivo.

Cuando la altura esté expresada en pisos y de la aplicación de este porcentaje resulte una fracción de piso igual o mayor que 0,5 se permitirá la construcción de un piso más. En el caso que dicho Plan Regulador contemple edificación continua, y el Conjunto Armónico no ocupe una manzana completa, el aumento de altura regirá sólo para las edificaciones aisladas construidas por sobre la altura máxima de la edificación continua, y se aplicará a partir de la altura máxima fijada para la edificación continua por el Plan Regulador respectivo.

Artículo 2.6.10. En los conjuntos armónicos el aumento del coeficiente de constructibilidad no permite exceder las alturas máximas ya incrementadas por la aplicación del artículo 2.6.9. ni libera del cumplimiento de rasantes y distanciamientos establecidos por el respectivo Plan Regulador o a falta de éste o si no contiene norma en la materia, por esta Ordenanza, sin perjuicio de lo dispuesto en el artículo 2.6.11. de este mismo Capítulo.

Del mismo modo, en los conjuntos armónicos el aumento de altura no libera del cumplimiento del coeficiente de constructibilidad ya incrementado por la aplicación de los artículos 2.6.5., 2.6.6. y 2.6.7., ni de las rasantes y los distanciamientos establecidos por el respectivo Plan Regulador o a falta de éste o si no contiene norma en la materia, por esta Ordenanza, sin perjuicio de lo dispuesto en el artículo 2.6.11. de este mismo Capítulo. (...)

Artículo 2.6.15. Un proyecto acogido a Conjunto Armónico podrá dividirse en partes, siempre que los predios resultantes de la subdivisión no sean inferiores a $2.500 \mathrm{~m} 2$ y que cada parte cumpla individualmente con las normas del Título 4 de esta Ordenanza.

Asimismo, podrán aprobarse proyectos acogidos a Conjunto Armónico emplazados en 2 o más predios colindantes, siempre que el terreno total involucrado cumpla las disposiciones del artículo 2.6.4. de esta Ordenanza y la solicitud de permiso sea suscrita por los propietarios de los predios involucrados.

En el expediente se deberá acompañar un plano de emplazamiento en que se grafiquen, a una escala adecuada, los predios y las edificaciones a desarrollar en cada una de las partes del proyecto y las normas urbanísticas de cada predio.

En caso que se contemplen servidumbres de paso entre los distintos predios éstas deberán señalarse en el proyecto.

Las normas urbanísticas se aplicarán a los proyectos de que trata este artículo como si se tratara de un solo predio, y en el caso que el predio o los predios queden afecto a dos o más zonas o subzonas del instrumento de planificación territorial, se aplicará lo preceptuado en el artículo 2.1.21. de esta Ordenanza. 
En caso de aprobarse un proyecto acogido a Conjunto Armónico emplazado en dos o más predios, el Director de Obras Municipales dictará la resolución del permiso de edificación acogiendo dicho proyecto a Conjunto Armónico. En estos casos, para efectos del control de las normas urbanísticas utilizadas por el total del proyecto, la Dirección de Obras Municipales deberá llevar un registro separado de los proyectos que se aprueben en conformidad al presente artículo.

Artículo 2.6.16. A los proyectos acogidos a Conjunto Armónico que se emplacen en dos o más predios, no les serán aplicables las normas sobre rasantes y distanciamientos en los deslindes entre predios del mismo proyecto.

Asimismo dichos proyectos podrán redistribuir la capacidad máxima de edificación de cada predio en el total del proyecto, siempre que el volumen total del proyecto no supere la suma de las capacidades máximas de edificación de los predios individuales y la altura máxima que resulte de la aplicación del artículo 2.6.9.

Las modificaciones de los proyectos a que se refiere el inciso primero de este artículo, relativas al emplazamiento, la volumetría o las fachadas de la edificación, requerirán que se acompañe a la solicitud de modificación la aceptación expresa de los propietarios de los demás predios, aun cuando tales modificaciones afecten sólo a la parte del proyecto emplazada en un solo predio.

Artículo 2.6.17. Los proyectos acogidos a la Ley No 19.537, sobre copropiedad inmobiliaria, clasificados como condominios Tipo B, deberán contemplar superficies de terreno de dominio común destinadas a vialidad, áreas verdes y equipamiento como si se trataran de proyectos de loteo.

En estos casos, el proyecto deberá asimilar los anchos de las vías conforme a la tipología que establece el artículo 2.3.4. de este mismo Título y el pavimento de las mismas se ejecutará conforme a las especificaciones mínimas que señalan los incisos quinto y siguientes del artículo 3.2.5., las cuales podrán ser modificadas por el arquitecto del proyecto, siempre que se aseguren condiciones similares en cuanto a resistencia y durabilidad.

Tratándose de proyectos clasificados como condominios Tipo A, los estándares y condiciones de diseño de las obras interiores de carácter colectivo, tales como vías de acceso, obras de pavimentación y áreas verdes, serán determinados por el arquitecto del proyecto.

Las obras de pavimentación señaladas no requerirán permisos especiales o la aprobación de un proyecto de las mismas, y serán recepcionadas por la Dirección de Obras Municipales bajo el carácter de obras complementarias.

Artículo 2.6.18. En la tramitación de solicitudes de proyectos acogidos a Conjunto Armónico, sólo le corresponde intervenir al Director de Obras Municipales, quien verificará como parte del proceso normal de revisión que el proyecto cumple las condiciones para acogerse a tales disposiciones, todo ello sin perjuicio de las revisiones que, en su caso, correspondan a los revisores independientes.

Artículo 2.6.19. El incremento de altura señalado en el artículo 2.6.9. de esta Ordenanza para los proyectos acogidos a Conjunto Armónico, podrá ser disminui- 
do en un $50 \%$ por los planes reguladores comunales, de acuerdo a las características de las zonas o subzonas de la comuna.

Asimismo, el plan regulador comunal podrá determinar zonas o subzonas en las que se rebajen hasta en un $50 \%$ los porcentajes de mayor constructibilidad señalados en los artículos 2.6.5., 2.6.6. y 2.6.7. IEURE

\section{Nota de agradecimiento}

Este artículo ha sido posible gracias a la colaboración de los arquitectos Amador Brieva, Germán Bannen, Rodrigo Rojas y Pedro Murtinho; los ayudantes de investigación Celeste Pottstock, Alexiss Machado de la Escuela de Arquitectura de la Universidad Andrés Bello; y los alumnos del curso Normativa Urbana y Forma Construida (2009), de la Escuela de Arquitectura de la PUC. El capítulo 3.3 del artículo fue elaborado a partir de las investigaciones: FONDECYT Iniciación No. 11090407 , Producción privada de espacios de uso público. Los casos resultantes de la normativa de incentivo de Providencia entre los años 1976 y 2006. Investigadora Responsable: Elke Elisabeth Schlack Fuhrmann. Investigación en curso 2010-2011. Así como DI 44-09/JM: La gestión de espacios de uso público en la planificación comunal: El Plan de espacios de uso público en la comuna de Providencia. Investigadora Responsable: Elke Elisabeth Schlack Fuhrmann. Investigación 2008-2010. 